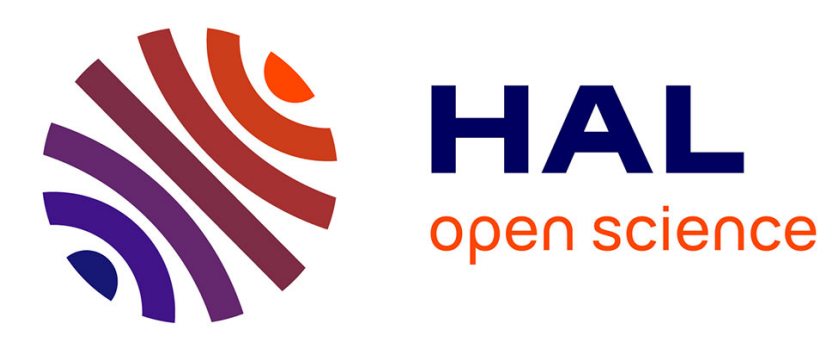

\title{
Numerical Assessment of Leading- and Trailing-Edge Control on a Swept Lambda Wing
}

\author{
Magnus Tormalm, Jean-François Le Roy, Stephane Morgand
}

\section{To cite this version:}

Magnus Tormalm, Jean-François Le Roy, Stephane Morgand. Numerical Assessment of Leadingand Trailing-Edge Control on a Swept Lambda Wing. Journal of Aircraft, 2018, 55 (2), pp.603-622. 10.2514/1.C033820 . hal-02382423

\section{HAL Id: hal-02382423 \\ https://hal.science/hal-02382423}

Submitted on 26 May 2021

HAL is a multi-disciplinary open access archive for the deposit and dissemination of scientific research documents, whether they are published or not. The documents may come from teaching and research institutions in France or abroad, or from public or private research centers.
L'archive ouverte pluridisciplinaire HAL, est destinée au dépôt et à la diffusion de documents scientifiques de niveau recherche, publiés ou non, émanant des établissements d'enseignement et de recherche français ou étrangers, des laboratoires publics ou privés. 


\title{
Numerical Assessment of Leading- and Trailing-Edge Control on a Swept Lambda Wing
}

\author{
Magnus Tormalm* \\ Swedish Defence Research Agency (FOI), SE-16490 Stockholm, Sweden \\ and \\ Jean-François Le Roy ${ }^{\dagger}$ and Stephane Morgand \\ ONERA-The French Aerospace Lab, FR-59045 Lille, France
}

\begin{abstract}
The next generation of agile unmanned combat aerial vehicles has put focus on the stability and control of low observable platforms. Without the traditional fin for yaw control combined with delta wings, a challenging task has been presented to the research community. The NATO research group AVT-201 was formed to meet this challenge and build on previous knowledge gained on the complex vortical flow of rounded leading-edge delta wings. This paper presents two numerical studies made by the Swedish Defence Research Agency (FOI) and the French Aerospace Lab (ONERA) on traditional trailing-edge control and a more unconventional type of leading-edge flap control. Results from the FOI-developed flow solver Edge, using trailing-edge control, on a low observable 53 deg swept lambda wing model are compared to experimental data. The effect of leading-edge control is investigated by ONERA with a slightly smaller-scaled model, using the in-house code elsA, and validated by experimental data. Additional static and dynamic derivatives are evaluated by ONERA for the clean configuration.
\end{abstract}

\section{Nomenclature}

wingspan, $\mathrm{m}$

root chord, $m$

reference length, $m$

frequency, $\mathrm{Hz}$

Mach number

static pressure, $\mathrm{N} / \mathrm{m}^{2}$

reference area, $\mathrm{m}^{2}$

static temperature, $\mathrm{K}$

velocity, $\mathrm{m} / \mathrm{s}$

angle of attack, deg

time step, s

static density, $\mathrm{kg} / \mathrm{m}^{3}$

freestream condition roll moment coefficient; $M_{x} /\left(q_{\infty} \cdot S_{\text {ref }} b / 2\right)$

pitch moment coefficient; $M_{y} /\left(q_{\infty} \cdot S_{\text {ref }} c_{\text {ref }}\right)$

yaw moment coefficient; $M_{z} /\left(q_{\infty} \cdot S_{\text {ref }} b / 2\right)$

side force coefficient; $F_{y} /\left(q_{\infty} \cdot S_{\text {ref }}\right)$

drag coefficient; $D /\left(q_{\infty} \cdot S_{\text {ref }}\right)$

lift coefficient; $L /\left(q_{\infty} \cdot S_{\text {ref }}\right)$

pressure coefficient; $\left(P-P_{\infty}\right) / q_{\infty}$

reduced frequency; $f \cdot c / V$

nondimensional angular velocities

dynamic pressure; $0.5 \cdot \rho \cdot \mathrm{V}^{2}, \mathrm{~N} / \mathrm{m}^{2}$

Reynolds number based on $c_{r}$

chordwise coordinate, body axis, $\mathrm{m}$

spanwise coordinate, body axis, $\mathrm{m}$

dimensionless wall distance

vertical coordinate, body axis, $m$

angle between velocity and rotation axes

dynamic viscosity, $(\mathrm{N} \cdot \mathrm{s}) / \mathrm{m}^{2}$

roll, pitch, and yaw angles

rotation rate on rotary balance

\footnotetext{
*Senior Scientist, Division of Defence and Security, Systems and Technology; magnus.tormalm@foi.se.

${ }^{\dagger}$ Engineer, ONERA-DAAP/MMHD; jean-francois.le_roy@onera.fr.

‡ONERA-DAAP/MMHD; stephanemorgand@ @ neuf.fr.
}

\section{Introduction}

$\mathbf{T}$ HE next generation of unmanned combat aerial vehicles (UCAVs), such as the Neuron (Fig. 1), emphasizes lowsignature, high-speed, and agile maneuvering. Without a traditional fin for yaw control and an inherent unstable configuration, the design of the flight control system will be critical. Accurate estimations of control effectiveness and dynamics are essential. It is believed, within the research community, that the advancement in computational fluid dynamics (CFD) and the ever-increasing computational resources have enabled a capability to predict these complex flows. The vortical flow associated with delta wings at a high angle of attack, in combination with deflected control surfaces, is still demanding. To meet this challenge, a NATO Science and Technology Organization (STO) task group was formed in 2011: Applied Vehicle Technology (AVT)-201, titled "Extended Assessment of Reliable Stability and Control Prediction Methods for NATO Air Vehicles." This group directly follows the successful work by AVT-113 [1-7] and AVT-161 [8-17] on basic aerodynamics, both static and dynamic, for generic delta wings, as well as a lambda wing planform. A blended-wingbody model was developed within the AVT-161 task group and was named the stability and control configuration, or SACCON.

The complex vortical flow of the SACCON is characterized by the formation of multiple leading-edge vortices: one pair originating from the apex, and another downstream at the tip region around an 8$10 \mathrm{deg}$ angle of attack (AOA). It is a consequence of the planform sweep and a variation of the leading-edge (LE) radius along the span. As the AOA is increased, the vortical systems travel toward each other and eventually meet at the midsection after inducing a third vortex around $\mathrm{AOA}=15 \mathrm{deg}$. It results in a distinct pitch disturbance. Controlling or reducing this lateral instability is the purpose of ONERA-The French Aerospace Lab (hereafter referred to as ONERA) study with unconventional LE mechanical devices.

This paper presents numerical results by the Swedish Defence Research Agency (FOI) and ONERA using respective in-house CFD solvers Edge and elsA. Both have studied the same SACCON geometry but at different model scales. FOI used the DLR-F19 model (Fig. 2) with a span of $1.538 \mathrm{~m}$, compared to the ONERA model span of $1.0 \mathrm{~m}$ (Fig. 2). Both have investigated the baseline geometry without control deflections but with different sting arrangements. 


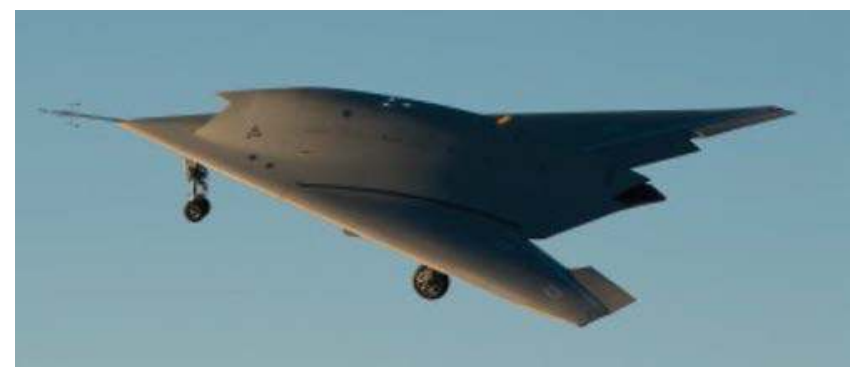

Fig. 1 First flight of Dassault-Saab Neuron in December 2012.

FOI included the 15 deg belly-sting mount, whereas ONERA did not model the rear sting. Longitudinal control was estimated with Reynolds-averaged Navier-Stokes (RANS) calculations by FOI looking at three cases: full roll deflections $\pm 20 \mathrm{deg}$ ) of both inner and outer trailing-edge control (case 1103), a case with only the outer controls deflected (case 1114), and a case with controls deflected on only one side (case 1144). The first two cases are defined as common within the AVT-201 group. ONERA has used RANS to assess the effect of the leading-edge device and compared it to the experimental data. Time-dependent dynamic simulations have also been done by ONERA to estimate the effect of the variation of the rotational point of the model during an oscillatory test.

\section{SACCON Geometry Description}

The SACCON geometry, shown in Fig. 3, was originally created by DLR, German Aerospace Center (DLR) and European Aeronautic Defence and Space Company-Military Air Systems (EADS-MAS) during the AVT-161 project. The planform features a lambda type of wing with a leading-edge sweep of $53 \mathrm{deg}$. The cranked trailing edges are swept \pm 53 deg to minimize reflecting angles. The wind-tunnel models have replaceable leading edges, enabling the testing of different sharp and rounded profiles. For the present study, we have only looked at the common test configuration featuring a complex variation of radius along the span. It starts with a sharp leading edge, at the apex or root chord, transforming into a round LE with a maximum radius at approximately the intersection between the inner body and outer wing, which declines to a minimal radius toward the wingtip. The trailing edges (TEs) are all blunt. For the DLR-F19 model, the thickness of the TE is approximately $2 \mathrm{~mm}$ at the root section and $1 \mathrm{~mm}$ at the wingtip. The root wing profile is fairly thick to allow for a realistic space for engines and weapon payload. From the intersection between the inner and outer body-wing, the wing is linearly twisted around the leading edge up to $5 \mathrm{deg}$ at the wingtip to improve aerodynamic behavior.

For the follow-on task (AVT-201), new wind-tunnel models were developed to incorporate different types of control surfaces. The DLR-F19 was equipped with four individual trailing-edge controls as well as a number of more unconventional types. Within this numerical study, FOI limited the investigation to TE controls. The size of the DLR-F19 model was determined by the load limits of the available model positioning mechanism in the German-Dutch Wind Tunnels (DNW) Niedergeschwindigkeits-Windkanal Braunschweig (NWB) low-speed wind-tunnel Braunschweig, shown in Fig. 2. The model was attached with a 15 deg cranked belly sting. Pressure ports were located at six stations, at 20 and $45 \%$ of the root chord (denotet $\mathrm{XC} 20$ and XC45 in Fig. 3), and cut perpendicular to the leading edge at 67 and 89\% (denoted LH67 and LH89 on the left side and RH67 and RH89 on the right side of the wing in Fig. 3). No pressure ports were located on the controls. A number of high-frequency Kulite pressure sensors were also installed on the DLR-F19 model. Control defections TE upward were defined as negative and downward as positive as shown in Fig. 5. The individual control surfaces were designated as left outboard (LOB) and left inboard (LIB), and they were designated similarly for the right side when looking from the top of the model [right outboard (ROB) and right inboard (RIB)].

The ONERA model has a smaller wingspan of $1.0 \mathrm{~m}$ and is optimized for the low-speed ONERA L1 and rotary balance SV4 tunnel in Lille. The model is made of carbon fiber and equipped with 96 pressure taps for static pressure measurements. The pressure taps are
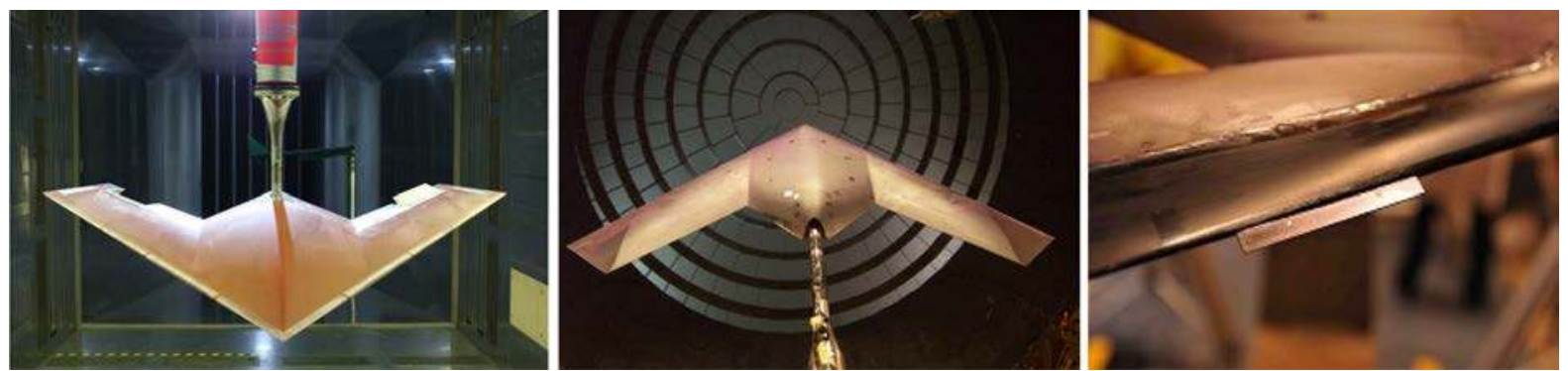

Fig. 2 SACCON model belly-mounted upside down in DNW-NWB low-speed wind tunnel (left), model in ONERA-L1 (middle), and details of the leading-edge slat (right).
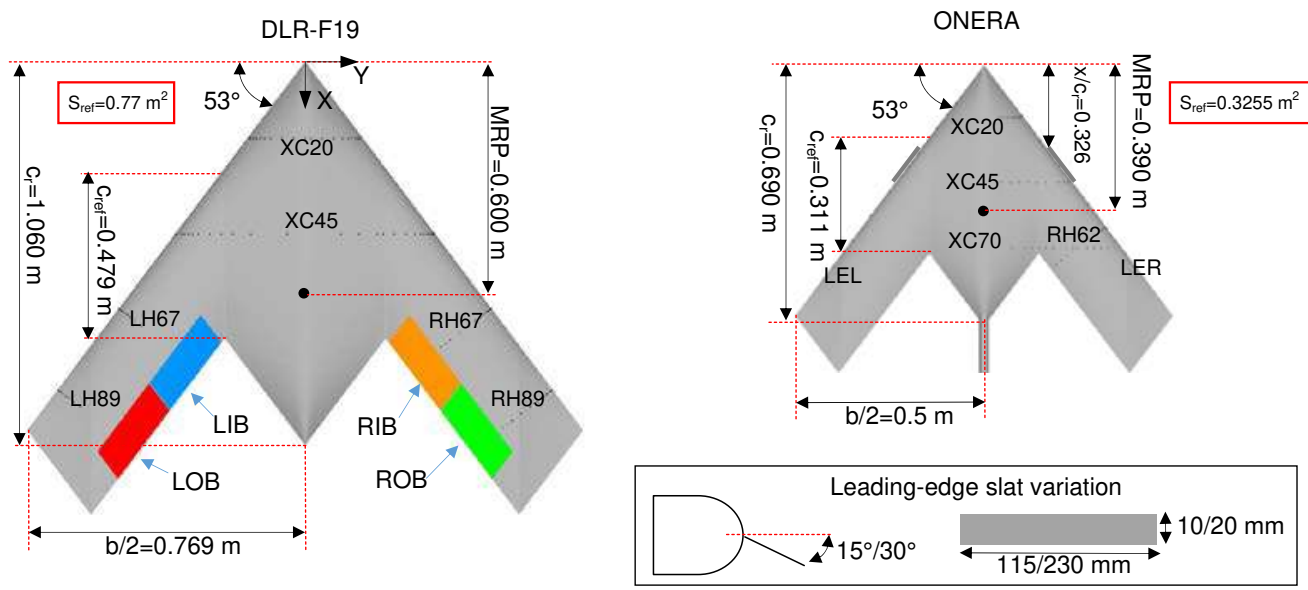

Fig. 3 Planform of the SACCON geometry comparing the DLR-F19 and ONERA models. 

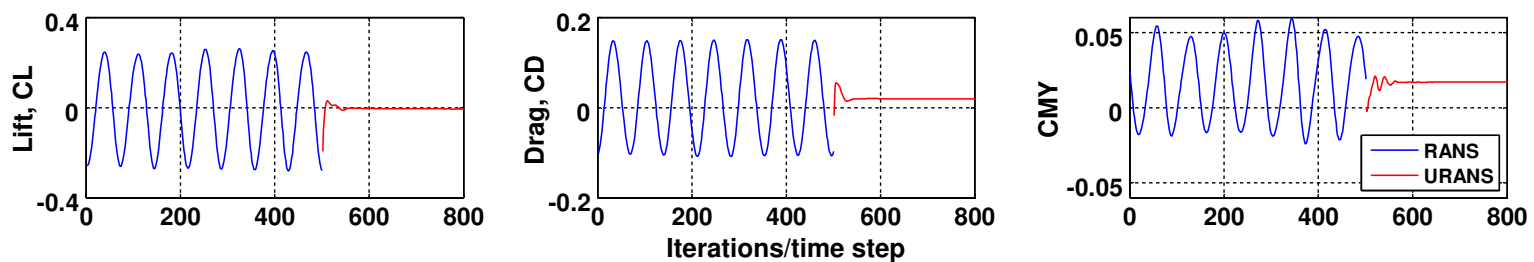

Fig. 4 FOI iteration history of force and moments at AOA $=0$ deg (case 1103) when switching from steady-state to time-accurate RANS. (URANS denotes unsteady RANS.)

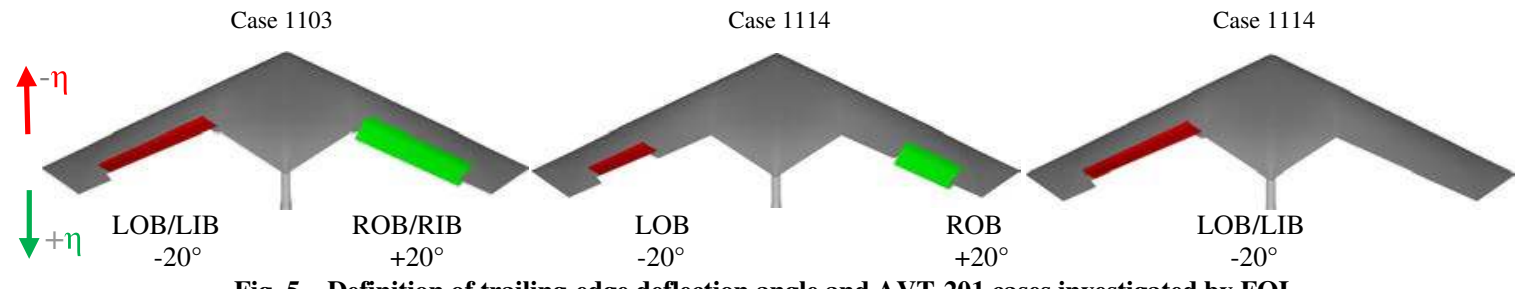

Fig. 5 Definition of trailing-edge deflection angle and AVT-201 cases investigated by FOI.

located at 20, 45, and 70\% (XC20, XC45 and XC70 in Fig. 3) of the root chord on the upper side of the starboard wing, as well as one row perpendicular to the LE at $62 \%$ (RH62 in Fig. 3). The ONERA model is also instrumented with pressure measurements along the leading edge (denoted LEL for the leding-edge left side and LER for the leadingedge right side). However, the pressure taps were not used for the campaign dedicated to LE control. The force and moments were measured using an internal six-component balance attached to a straight sting. Transition tripping was fixed using $0.18 \mathrm{~mm}$ dots at a constant distance of $11.5 \mathrm{~mm}$ (3.7\% of the wing chord) on the upper side for the reference tests. Transition tripping was removed for the test with LE control. The effectiveness of the transition triggering at the upper side was validated by thermal measurements [18] for a low AOA.

The ONERA model could be fitted with slats at various positions on the leading edge between $2 y / b=0.29$ and $2 y / b=0.75$. The slats tested were flat $1.2 \mathrm{~mm}$ rectangular plates with a width of $10 / 20 \mathrm{~mm}$, a length of 115 or $230 \mathrm{~mm}$ (Fig. 3), and an angular setting of 15 or 30 deg downward. Both sides of the wing leading edges were fitted with slats.

The moment reference points (MRPs) on both models were specified at the same relative location of the root chord at $x / c_{r}=$ 0.566 from the apex. Additional reference data used in the numerical analysis for both FOI and ONERA are specified in Fig. 3. Lateral and longitudinal controls (yaw and rolling moments) were normalized with the half-span dimension. The coordinate system used throughout this paper was located with its origin at the apex of the model. The $x$ axis was pointing aft, the $y$-axis was pointing to the right side when looking from behind and the $z$-axis was pointing upwards according to the right-hand rule.

\section{Computational Method}

FOI-developed code Edge [19] is a three-dimensional Reynoldsaveraged Navier-Stokes solver for compressible flow on unstructured grids. The solver has an edge-based formulation and uses node-centered finite volume techniques. The edge-based formulation makes it possible to compute any type of element: structured or unstructured. The control volumes are non-overlapping and are formed by a dual grid, which is computed by the preprocessor from the control surfaces for each edge of the primary input mesh. Edge can be used for both steady-state (SS) and time-accurate (TA) calculations. Time-accurate computations can be performed using a semi-implicit dual-time-stepping scheme that exploits a convergence acceleration technique via a steady-state inner iteration procedure. A large number of turbulence models are available, which are categorized into three different groups: RANS, detached-eddy simulation or hybrid RANS/large-eddy simulation, as well as largeeddy simulation. Edge is primarily developed by FOI, but several universities and research institutes have joined the Edge community and contribute to its advancement. Edge is the main aerodynamic tool used by FOI and Saab.

All FOI cases were initially run in steady-state RANS mode with Edge, version 5.2 (revision 3115). Steady-state time integration was achieved with a three-stage Runge-Kutta scheme. The spatial discretization used a second-order central scheme with a Jameson type of artificial dissipation, with coefficients set to 0.5 and 0.02 . The primary turbulence model was the Wallin and Johansson [20] explicit algebraic Reynolds stress model (EARSM) with the Hellsten [21] $k-\omega$ model. The solid surface was modeled using an adiabatic weak wall boundary condition assuming fully turbulent flow. Actual windtunnel data on the static pressure, temperature, and freestream velocity were set at the far-field boundary with a weak characteristic formulation. Time histories of the individual boundaries were recorded; otherwise, Edge 5.2 default input values were used.

The steady-state RANS approach turned out to show low convergence, leveling out with highly oscillating behavior, as shown in Fig. 4. The surface Cp plots looked unstable, even at low AOAs. It was therefore decided to run a selected number of AOAs with a timeaccurate RANS approach. The dual-time scheme was setup with an implicit time step of $1 \mathrm{e}-4 \mathrm{~s}$ and 50 inner iterations to reach an inner convergence by at least one order of magnitude.

The time-accurate RANS cases were initialized from the final steady-state RANS solutions and run for a limited 300 time steps, with mean value sampling starting after 150 time steps. The total accumulated time was $0.03 \mathrm{~s}$, corresponding to 1.5 flow passes of the root chord. Additional time and budget would have resulted in additional time steps but, as shown in Fig. 4, the force and moment stabilized dramatically with the time-accurate RANS approach and values were leveling out after only 100 steps. Similar behavior was seen for all time-accurate cases, also seen for time-accurate cases at high AOA.

Edge is a fully parallelized code using the open message-passing interface for communication. FOI RANS computations were run on a three-level full multigrid cycle of $500+500+5000$ iterations, which took approximately $14 \mathrm{~s} /$ it with 64 cores on FOI's J29 Linux cluster.

All ONERA computations were conducted using the in-house Navier-Stokes flow solver elsA [22], which stands for ensemble logiciel pour la simulation en aérodynamique (Software package for aerodynamic simulations). It is a software package based on objectoriented solutions for fluid dynamics numerical simulation, created by combining the $\mathrm{C}++$ language, for translating object concepts with the Fortran language to implement time-costly scientific computation methods. Python, a public domain interpreted object-oriented language, is used for the interface.

elsA is a cell-centered finite volume CFD code that solves the Navier-Stokes equations on block-structured grids. Several turbulence models are implemented, including algebraic, one-equation, twoequation, and five-equation models. A transition prediction model is 
also implemented. All ONERA computations have been carried out in a "fully turbulent" mode using the Spalart-Allmaras one-equation turbulence model.

To take into account the rotation of the computational domain for dynamic derivative predictions, an arbitrary Lagrangian-Euler formulation was used $[23,24]$. The grid velocity only resulted from the composition of a motion of translation and rotation, and there was no time-varying cell volume. In the Navier-Stokes equations, the grid motion was taken into account by the rigid-body technique developed in the elsA software and applied to rotorcraft simulations. The mesh was moving but not deforming. ONERA used the moment formulation technique, which allowed the freestream conservation property to be respected. This technique replaced the classic geometric conservation law, which would be more expensive and less accurate in this case. The input parameters of the motion were the center and the axis of rotation, the amplitude, and the frequency.

To solve the time-accurate flowfield, the dual-time-stepping (DTS) method is used. The DTS method allows the resolution of unsteady problems using subiterations between two physical times. Thus, it belongs to the family of the subiterative methods and resorts to the introduction of a fictitious time step called dual time, which is contrary to other methods that give preference to the use of physical time. Calculations between the two physical times lead to the resolution of a system of pseudounsteady equations in dual time and are carried out using convergence acceleration techniques developed within the framework of the steady problems in the elsA software.

For the steady-state computations, the set of ordinary differential equations in time is solved by means of the backward Euler scheme. An implicit phase is used for the convective terms. The viscous terms are treated with a lower-upper factorization. The resolution of the turbulence equation is not coupled to the others, and the Harten's parameter is fixed to a small value $(0.01)$ to achieve a good accuracy.

Jameson's central scheme has been used for the discretization in space of ordinary differential equations in these simulations. The two parameters of artificial viscosity, $k i 2$ and $k i 4$, associated with this scheme are set to low values of 0.0 and 0.016 , respectively, to minimize the damping effects. This scheme is well adapted for the computations at low speed.

A sensitivity study for some discretization parameters has been carried out: various combinations of preconditioning and dissipation factors were tested and are discussed in detail in the thesis [18] by Morgand. These parameters have a noticeable effect on the pitching moment values or on the numerical convergence.

\section{Computational Meshes}

Four geometrical configurations have been analyzed by FOI, and three have been analyzed by ONERA. Both FOI and ONERA have calculated the baseline clean reference case without any control deflections but at different model scales and with different sting arrangements. FOI has generated full body meshes for all cases: the reference (1001), and the three roll control cases (see Fig. 5). The maximum roll case is designated "1103" in the AVT-201 group. Case 1103 has both TE controls on the left side deflected upwards and both TE control on the right side deflected downwards. FOI has also calculated two additional roll cases: one with only the outer surfaces deflected (1114), and one with controls deflected on the left side only (1144). The deflection angle has been $\pm 20 \mathrm{deg}$ in all cases. FOI has included the $15 \mathrm{deg}$ cranked belly sting and has closed all gaps around the controls. The gaps were on the order of $1 \mathrm{~mm}$ in the original CATIA Version 5 geometry.

FOI used a two-step approach to generate the meshes. In the first step, the ANSYS ${ }^{\circledR}$ ICEMCFD $^{\text {TM }}$ tetra octree approach was used to build the surface mesh and an inviscid background mesh. The far field was modeled as a square box, $500 \mathrm{~m}$ away in all directions. The blunt trailing edges were coarsely resolved with one to four elements. More care was taken at the leading edges with very small cell sizes. The offbody volume above and behind the model was refined using density boxes (Fig. 6b). In the second step, FOI mesh generator TRITET [25] was used to grow the prismatic boundary layers and the final inner volume mesh. A newly implemented smoothing routine for the background grid allowed for a better-quality mesh. The predominantly octree type of volume mesh generated by ICEMCFD Tetra suffered from large volumetric changes. The smoothing in TRITET is limited to the volume and could not be used on the surface. TRITET built the prismatic boundary layer normal to the walls from the ICEMCFD surface mesh. The number of added layers depended on user input or the element size of the inviscid background mesh. TRITET stopped adding prismatic elements in areas where the size matched that of the tetrahedra in the outer mesh to allow a smooth volumetric transition. The final inner volume was also generated in TRITET using the advancing front technique with sizes from the smoothed background mesh.

Several initial meshes were tested by FOI before the final setup with a highly refined LE resolution was fixed. The final meshes consisted of 30-35 million nodes, as listed in Table 1. The basic layout was similar between the four configurations; all used a maximum of 45 boundary layers with an initial height of $2 \mathrm{e}-6 \mathrm{~m}$ and an expansion ration between 1.20 and 1.22. A typical mean value of the first cell $y^{+}$was around 0.3, as shown in Fig. 6a. A typical size for the surface triangles at the LE was 0.15 to $0.25 \mathrm{~mm}$, and around $3 \mathrm{~mm}$ at the upper wing. The difference in node counts between the four FOI meshes was mainly due to the deflected controls that generated a complex prismatic layer due to splitting of the surfaces. This was solved by clustering nodes in the local areas of the split. The final mesh for one configuration is shown in Fig. 7.

The ONERA meshes were also based on the SACCON CAD geometry, as provided by DLR, with the rounded leading edge. The DLR geometry was scaled to a span of $1.0 \mathrm{~m}$. The computation grids generated by ONERA were multiblock structured and built with ICEMCFD Hexa software around the half-span. The outer boundary of the domain was specified as a large cylinder, with a radius of $50 \mathrm{~m}$ and length of $100 \mathrm{~m}$, in order to minimize the risk of reflection on the outer boundaries. The topology of these grids was $\mathrm{C}-\mathrm{H}$, filling the space between the model surface and the far field. The H topology

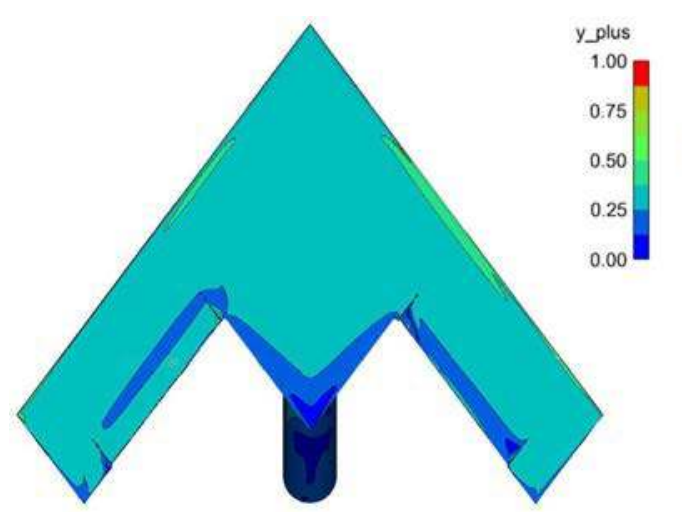

a) Mean $\mathrm{y}^{+}=0.306$ at $\mathrm{AOA}=0 \mathrm{deg}$

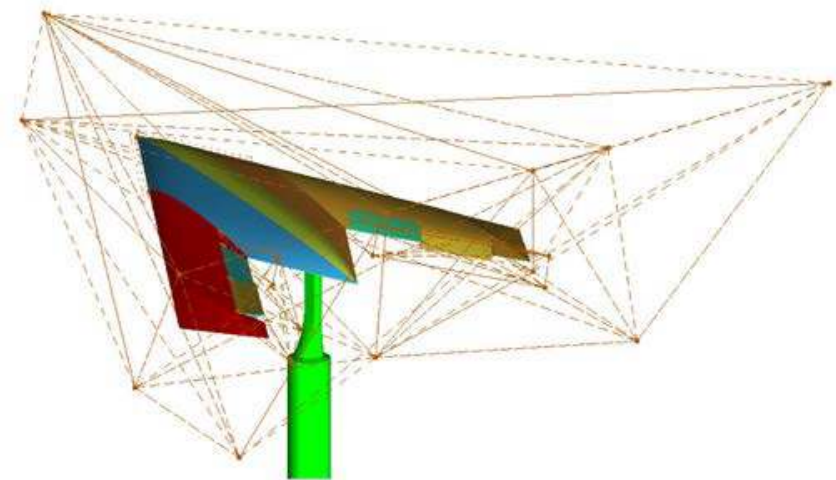

b) Density boxes in ICEMCFD around the model 
Table 1 Computational grid size and parameters

\begin{tabular}{|c|c|c|c|c|c|c|c|c|c|c|c|c|c|}
\hline & Span & Sting & Gaps & Nodes $10^{6}$ & Tetra $10^{6}$ & Prisms $10^{6}$ & Hexa $10^{6}$ & Surf. $10^{3}$ & $\mathrm{BL}$ & Initial, $\mathrm{mm}$ & Exp.ratio & Size at LE, $\mathrm{mm}$ & Size at wing, $\mathrm{mm}$ \\
\hline FOI, base & Full & Belly & No & 30.0 & 77.7 & 32.9 & -- & 1018.0 & 45 & $2 e-3$ & 1.20 & $0.15-0.25$ & 3 \\
\hline FOI, 1103 & Full & Belly & No & 34.7 & 93.5 & 37.0 & -- & 1137.4 & 45 & $2 e-3$ & 1.20 & $0.15-0.25$ & 3 \\
\hline FOI, 1114 & Full & Belly & No & 34.9 & 94.0 & 37.2 & -- & 1148.5 & 45 & $2 e-3$ & 1.20 & $0.15-0.25$ & 3 \\
\hline ONERA, mesh1 & Half & No & -- & 10 & -- & -- & 10 & 22.9 & 41 & $4 e-3$ & 1.10 & $4.5^{\mathrm{a}}$ & 10 \\
\hline ONERA, mesh2 & Half & No & -- & 15 & -- & -- & 15 & 51.5 & 41 & $4 e-3$ & 1.10 & $2.8^{\mathrm{a}}$ & 5.6 \\
\hline ONERA, mesh3 & Half & No & -- & 30 & -- & -- & 30 & 84.5 & 41 & $4 e-3$ & 1.10 & $2.5^{\mathrm{a}}$ & 5.4 \\
\hline
\end{tabular}

${ }^{a}$ Spanwise extent, where points were clustered chordwise.

Note: Hexa, hexahedral element; Surf., surface element; BL, boundary layers; Exp., exponential.

was preferred to better take into account the thickness of the trailing edge. A number of grid refinements was investigated by Morgand [18], but only three meshes are presented here. The mesh on the wing surface was defined, respectively, by 107,135 , and 207 points in the chordwise direction and 135, 225, and 245 points in the spanwise direction for the three meshes, called mesh1, mesh2, and mesh3. The height of the first cell was constant at $0.004 \mathrm{~mm}$ to get $y^{+}$close to one. The boundary layer was defined by 41 points. The refinements were concentrated in the vicinity of the leading edge, clustered in the chordwise direction. A typical element size at the middle of the wing was between 5 and $10 \mathrm{~mm}$. In these conditions, the coarse grid (named mesh1) contained 10 million points, the medium grid (mesh2) contained 15 million points, and the fine grid (mesh3) contained 30 million points for the half-span model. Figure 8 shows the finest ONERA surface mesh distribution of the half-span model (mesh3).

The ONERA configurations with mechanical LE slats were built using the chimera method. An additional grid around the slat and the leading edge of the UCAV was generated (Fig. 8). The assembly of the two grids was realized with special tools that manipulated the CFD General Notation System (CGNS) tree.
A summary of the grid size and the parameters for all meshes is found in Table 1.

\section{Results and Discussion}

\section{A. Flowfield Topology}

The low-speed high-AOA aerodynamics of the SACCON lambda wing planform is dominated by vortical flows. As with conventional delta wings [26,27], the flow separates at the sharp leading edge at a relatively low AOA. The resulting shear layer rolls up, and the flow reattaches to the model surface to form a vortex structure. The flow topology, based on particle image velocimetry (PIV) measurements as a function of the angle of attack, was presented in the thesis by Morgand [18]. Moreover, the vortical flowfield developing above the SACCON wing is mainly influenced by the specific shape of the LE and the spanwise variation of the radius, which causes the generation of multiple vortex systems. These vortices result in a nonlinear evolution of the aerodynamic coefficients at relatively low AOAs for a delta wing. One can divide the aerodynamic pitching moment behavior, as seen in Fig. 9c, into three regions: first, a mostly linear part below

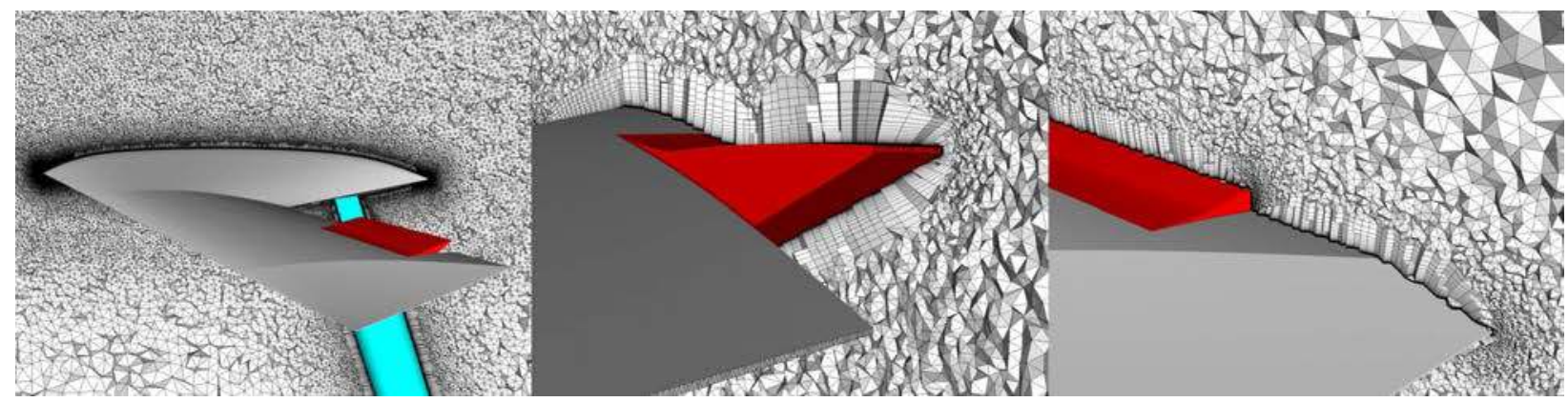

Fig. 7 FOI mesh at symmetry and across outer control of case 1114.
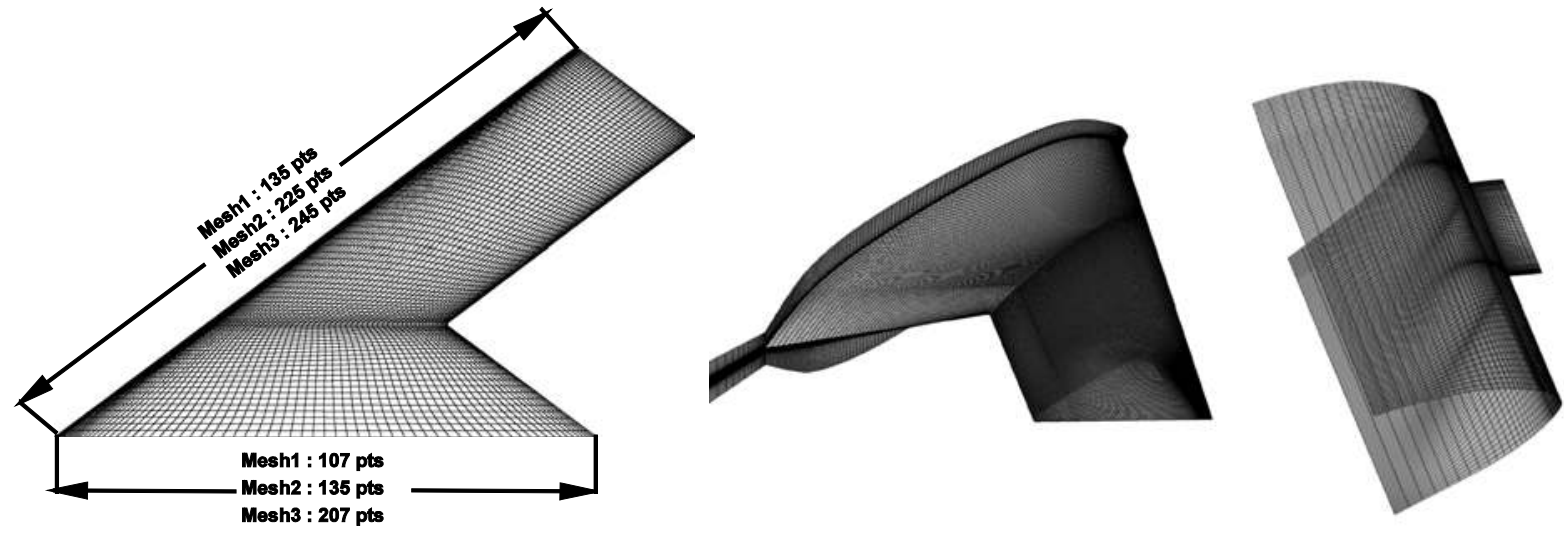

Fig. 8 ONERA surface mesh and part of symmetry plane mesh and detail of short LE slat. 
$\mathrm{AOA}=10 \mathrm{deg}$, and then a nonlinear domain including a pitchdown phenomena between 14 and $19 \mathrm{deg}$. Finally, at higher AOA, a peak is reached after which the moment drops again.

Below $\mathrm{AOA}=8 \mathrm{deg}$, the flow remains mostly attached around the wing. With increasing AOAs, considering each side of the wing, two vortices appear on the upper surface of the SACCON model: one upstream in the apex region, and the other downstream in the wingtip region. They are initiated where the radius is sharp, i.e., the rounded LE delays the flow separation to higher AOAs $[18,28]$. The formation of the tip vortex occurs around $\mathrm{AOA}=8 \mathrm{deg}$, and the apex vortex occurs just after at around $\mathrm{AOA}=9 \mathrm{deg}$. As the $\mathrm{AOA}$ is further increased, both the apex and tip vortex become stronger and start to move toward each other. The vortex-induced suction produces front and rear local lifts, which balance each other around the pitching MRP.

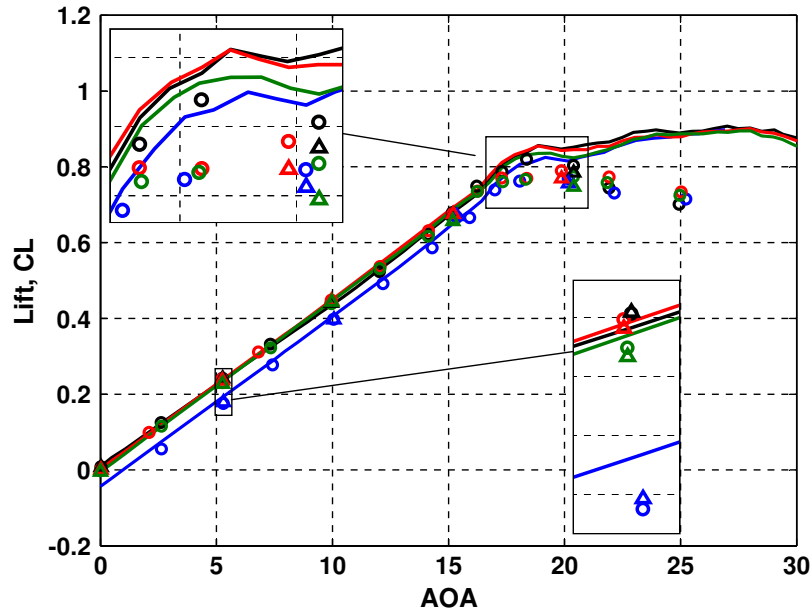

a) Lift

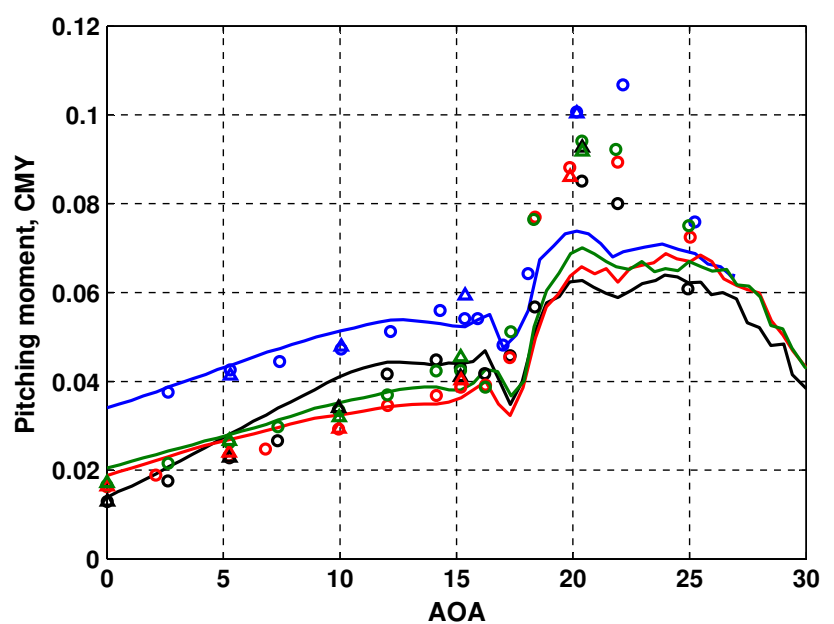

c) Pitching moment

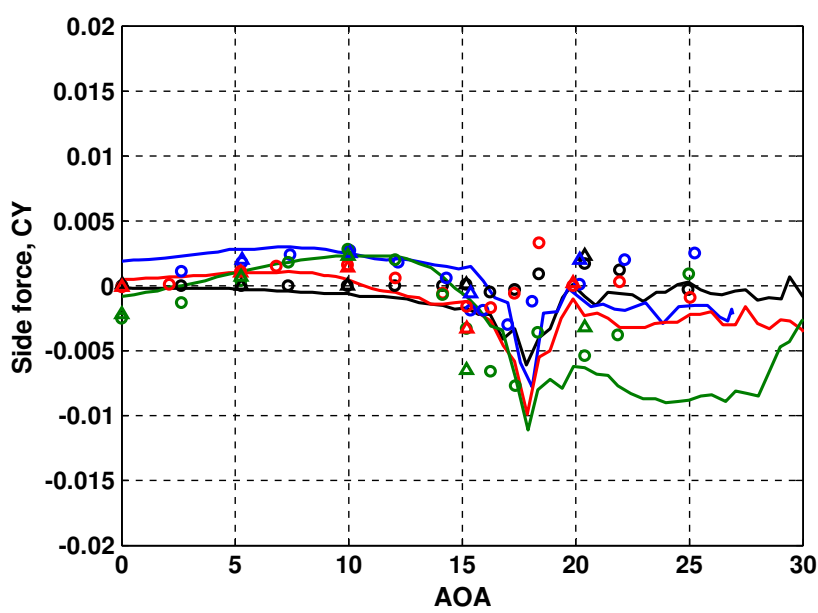

e) Side force

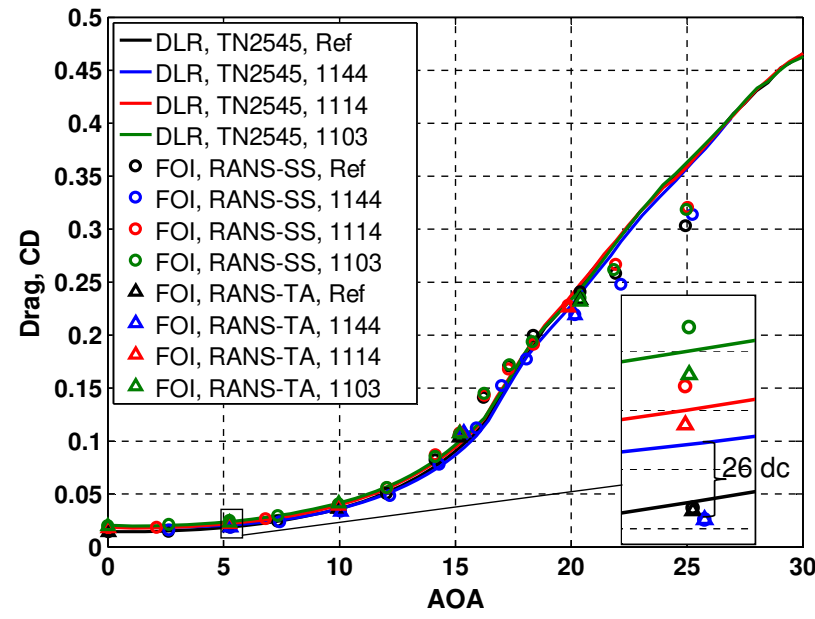

b) Drag

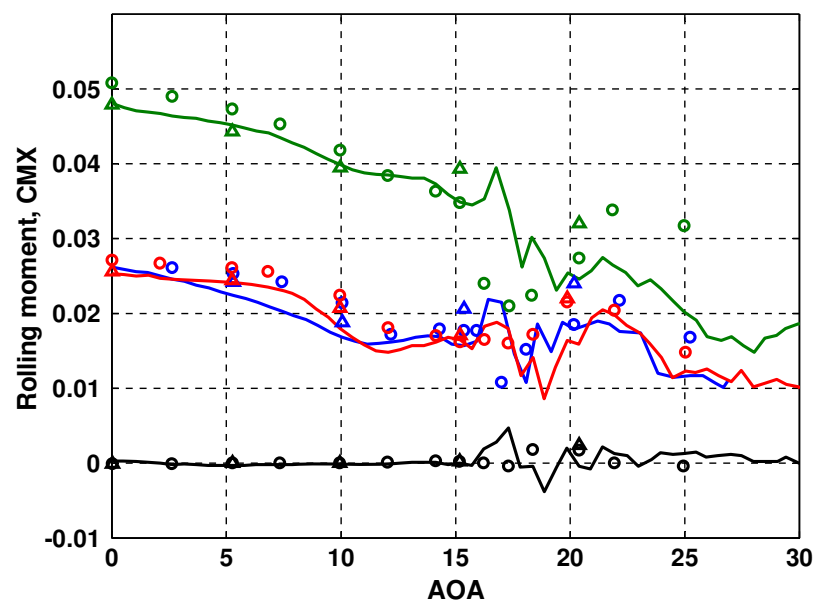

d) Rolling moment

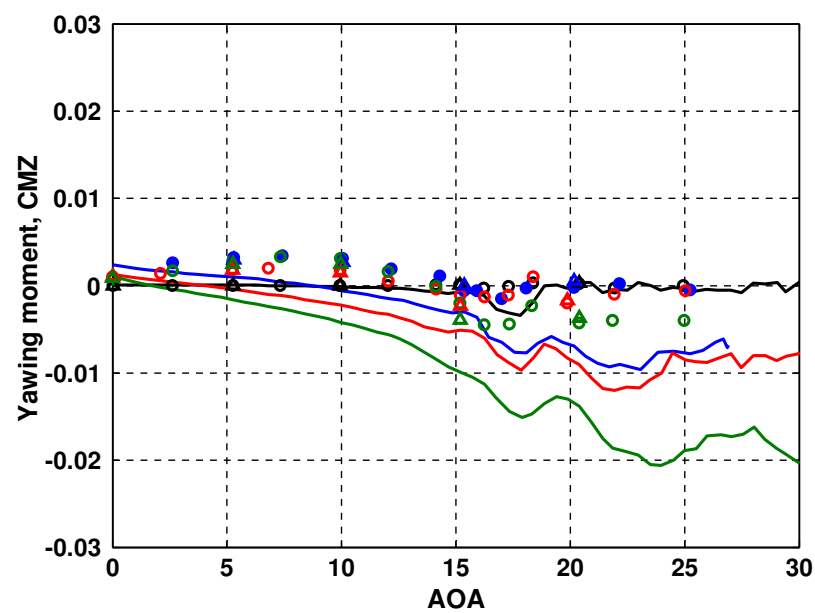

f) Yawing moment

Fig. 9 FOI integrated force and moment results of TE control: $M_{\infty}=0.15, R e=1.6 \cdot 10^{6}$. 
At 15-16 deg, in the PIV results [18] as well as the numerical investigation, a dramatic shift is seen when a third vortex can be identified appearing between the apex and the tip vortex. This vortex originates from a region of the LE section with large radius at about $x / c_{R}=0.28$. A shift of location for the downstream tip vortex is seen as it is drawn toward the middle and induces more low pressure on the rear part of the wing, causing the pitch down.

Above AOA $\sim 16 \mathrm{deg}$, the lowest point of the dip is reached and a nose-up effect is observed, due to a reduced strength of the tip vortex, but strong suction is still induced by the apex vortex in front of the MRP. After AOA $=17 \mathrm{deg}$, all vortical systems are more or less merged into one major system displaying a spiraling pattern (Fig. 14). The surface pressure plots at the apex reveal at least two separate lowpressure regions, indicating some separation. The suction on the aft part of the wing is further reduced, probably due to vortex breakdown moving upstream.

The experimental pitching moment peak is reached around $\mathrm{AOA}=25 \mathrm{deg}$. After this point, numerical simulations indicate the formation of an additional vortical system forming at the swept TE of the outer wing. It generates low surface pressure at the rear part of the upper wing again, and is thus responsible for the pitch down seen at the highest AOA.

\section{B. Integrated Forces and Moment Result of Reference Configuration}

The clean configuration, or reference case (Ref in Fig. 9), was evaluated by both FOI and ONERA. FOI force and moment integration was evaluated and stored at every iteration/time step for each individual boundary patch. Due to the oscillatory behavior using the steady-state RANS approach by FOI, mean values of the final 500 iterations were calculated and are presented here. For the timeaccurate RANS cases, mean values were calculated for the final 100 time steps of the 300 in total. It can be concluded from the results that the different setups produced values in close agreement. However, a nonconverged RANS solution should not be trusted, and no flowfield visualizations for these cases are therefore shown.

FOI cases are all calculated with actual wind-tunnel conditions. The nominal speed of the tunnel is $50 \mathrm{~m} / \mathrm{s}$, or a Mach number of 0.15 , corresponding to a Reynolds number of 1.6 million based on the reference chord. FOI lift predictions, shown in Fig. 9a, match well up to $\mathrm{AOA}=16 \mathrm{deg}$. Looking closer at the linear AOA range around $6 \mathrm{deg}$, the lift slope predictions are in close agreement with levels within 5\%. FOI predictions at the high-lift range are dropping faster with a lift peak around $\mathrm{AOA}=20 \mathrm{deg}$. The maximum lift around $\mathrm{AOA}=27 \mathrm{deg}$ found in the DLR-F19 experiment is not correctly predicted by the steady-state RANS method by Edge. The timeaccurate RANS simulation is only done in steps of $5 \mathrm{deg}$, up to $\mathrm{AOA}=20 \mathrm{deg}$, due to the higher computational cost.

FOI drag predictions in Fig. 9b are within five drag counts (dc) at the lower AOA range, except for case 1144, which is about 26 drag counts off. As with the lift, the drag is not so well predicted using SSRANS at the higher-AOA range. The pitching moment shows an overshoot above $\mathrm{AOA}=20 \mathrm{deg}$ for FOI cases. In a previous investigation by the AVT-161 group (reference case 1001), pitching moments comparable to the experiment could only be obtained after modeling the sting and wind-tunnel walls in a delayed detached-eddy simulation (DDES) shown by Jirasék and Cummings [29]. The tripping applied in the experiment is not directly modeled in the simulations. The computational approach results in higher-pressure peaks at the apex region compared to experimental data. Further enhancement of the mesh resolution and different turbulence models may provide results in closer agreement. It could be concluded that the prediction of separation at the rounded LE is difficult and is very sensitive to the methods used, both numerically and experimentally due to the tripping applied. Cases with deflected controls show a pitching moment prediction in better agreement with experimental data at the lower-AOA range but still result in an overshoot of pitching moment around $\mathrm{AOA}=20 \mathrm{deg}$.

The ONERA clean (reference) configuration result is shown in Fig. 10. The numerical simulations are, in general, in good agreement with the experimental data (overall shapes of the curves). The lift
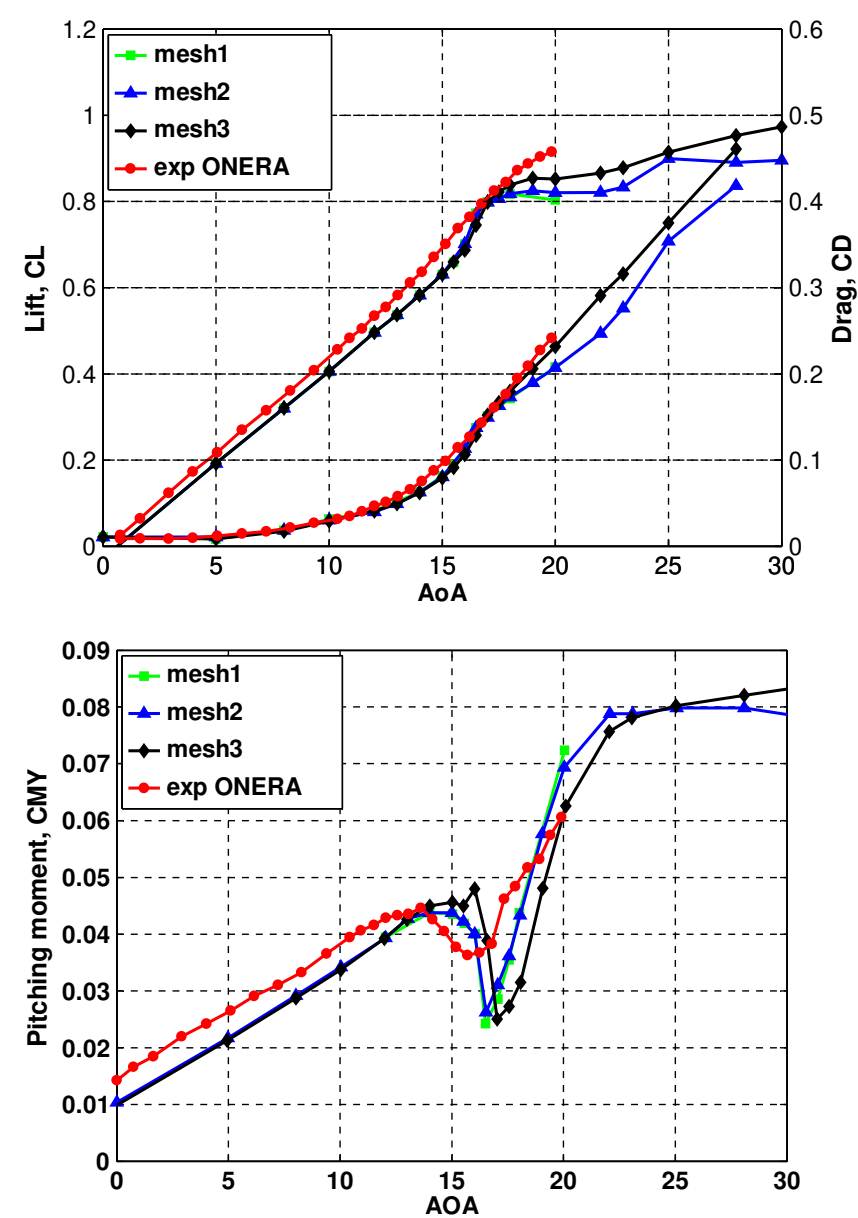

Fig. 10 ONERA experimental (exp) and numerical force and moment results of clean (reference) case comparing different mesh resolutions: $M_{\infty}=0.146, R e=1.13 \cdot 10^{6}$.

prediction using elsA shows a small offset at the lower linear AOA range without any visible difference between meshes up to $\mathrm{AOA}=15 \mathrm{deg}$. The mesh refinement results in some variations at higher AOAs without any maximum lift point reached. The computed pitching moment exhibits an offset in the linear range, and the pitching moment dip is appreciably deeper. The comparison of different mesh resolutions shows that even the coarse meshes predict the global behavior correctly. However, mesh refinement (mesh 3) further delays the dip of the pitching moment coefficient between 15 and $16 \mathrm{deg}$ of the AOA. The dip phenomenon is due to the onset of the third vortex. The nonlinear aerodynamic contribution of the vortices is well predicted.

\section{Evaluation of Trailing-Edge Control by FOI}

To be able to claim a controllable configuration, all three axes should be investigated regarding control efficiency. FOI investigation was limited to the longitudinal axis, thus only looking at roll control. As seen in Fig. 9d, the roll moment curves are well predicted for all configurations, both in levels and slopes. The time-accurate RANS does the best job and is closest to experimental data. The maximum roll efficiency is achieved with all four control surfaces deflected (case 1103). If only the outer controls (case 1114) or controls on just one side (case 1144) are applied, the control efficiency is around 50\% of maximum. The DLR-F19 model shows a dramatic diverging behavior above $15 \mathrm{deg}$. This can be seen in both the experimental and computational data. All moment curves display a highly nonlinear behavior, making flight unrealistic above $\mathrm{AOA}>15 \mathrm{deg}$.

The experimental side force shows an asymmetry for the baseline case, even if the forces are small. It may be due to some geometric 
deficiency on either the model or the measuring equipment. This could perhaps also explain the difference seen in the yawing moment. FOI yawing moment is initially predicted with a different sign and slope.

Figures 11 and 12 show the $\mathrm{Cp}$ sections for the different control surface configurations. The agreement with experimental data is good, with some differences found at $\mathrm{AOA}=15 \mathrm{deg}$ at the outer controls on the left side (LH89). The numerical data have a flat curve, indicating full separation; whereas the experimental shows higher values at the LE. Note that no experimental data on the actual control surface are available. Another difference is a stronger pressure peak at $x / c=0.2$ and $\mathrm{AOA}=20 \mathrm{deg}$ for the computational cases. This is probably the reason for the pitching moment overshoot. The experimental data have a single flatter and lower peak, which might be related to a more diffuse or integrated mix between the apex and trailingedge vortex after the merge around $\mathrm{AOA}=15 \mathrm{deg}$. Numerical data at $x / c=0.45$ and $\mathrm{AOA}=20 \mathrm{deg}$ indicate two pressure peaks, which are only supported by experimental data at lower AOAs.

The vortical flow topology is identified with vorticity and the $Q$ criterion. Figure 13 shows vorticity at every $\Delta x=0.1 \mathrm{~m}$ station. The vorticity is computed from $y$ - and $z$-velocity components using the built-in vorticity function in EnSight 9.2.2e. At a zero AOA, vortical structures are seen emerging from the tip of the controls when deflected. A small tip vortex is also found on the lower side due to
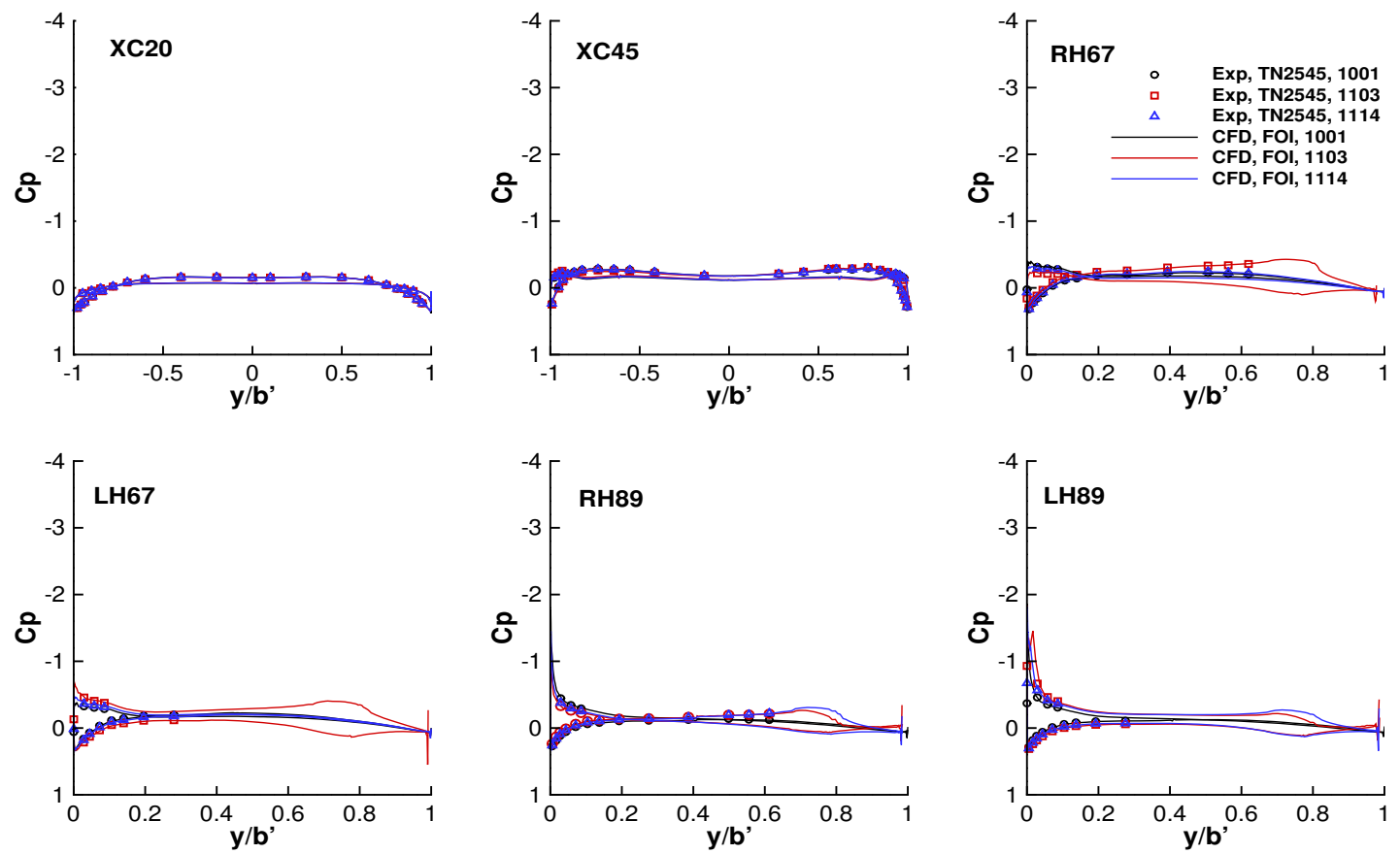

a) $\mathrm{AOA}=0^{\circ}$
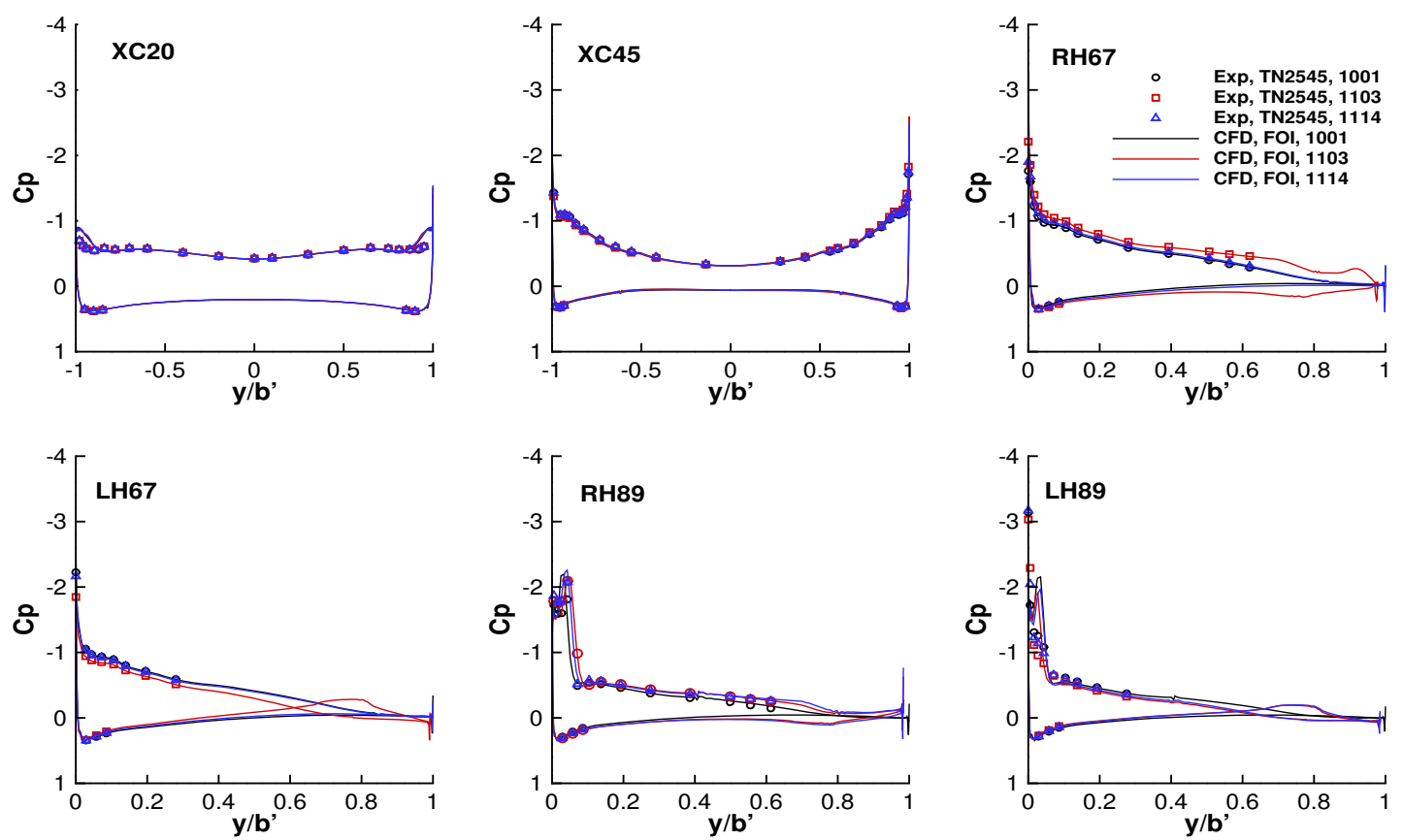

b) $\mathrm{AOA}=10^{\circ}$

Fig. 11 Cp sections comparing FOI time-accurate RANS and DLR experiments: $M_{\infty}=0.15, A O A=0$ and 10 deg, and $R e=1.6 \cdot 10^{6}$. 
the $5 \mathrm{deg}$ twist of the outer wing. Below $\mathrm{AOA}=10 \mathrm{deg}$, the different control cases show differences in the vortical flow due to the deflected TE controls. At 15 and $20 \mathrm{deg}$, the effect of the TE control cannot be separated from the tip and apex vortex that dominate the flow.

Another way of identifying the vortical structures is with $q$ criteria colored with helicity, as in Fig. 14. These figures were created with the available $Q$ criteria and relative helicity functions in EnSight 9.2.2e. The rotational direction of the vortices is revealed by the color (thus sign) for helicity. The fine mesh by FOI in the wake provides long isosurface structures of the $Q$ criteria.

Figures 15 and 16 show the upper surface pressure and streamlines based on skin friction. It is interesting to note how the streamlines develop as the AOA is increased: especially the surface flow at the trailing edge where the control surfaces are located. Already at $\mathrm{AOA}=5 \mathrm{deg}$, the surface flow starts to align with the trailing edge, thus reducing the efficiency of the controls. This may be the main reason why the controls seem to have little effect and a very nonlinear behavior above $\mathrm{AOA}=15 \mathrm{deg}$. The surface flow also indicates where the leading-edge vortices are located.

Figures 17-20 show volume cuts across the controls surface sections. If both controls are deflected downward (case 1103), the flow shows a separation already at AOA $=0 \mathrm{deg}$ for the RH89 cut. If only the outer control is deflected (case 1114), the situation improves. At higher AOAs, above $15 \mathrm{deg}$, the flow is fully separated, making the efficiency very low for these types of trailingedge controls.
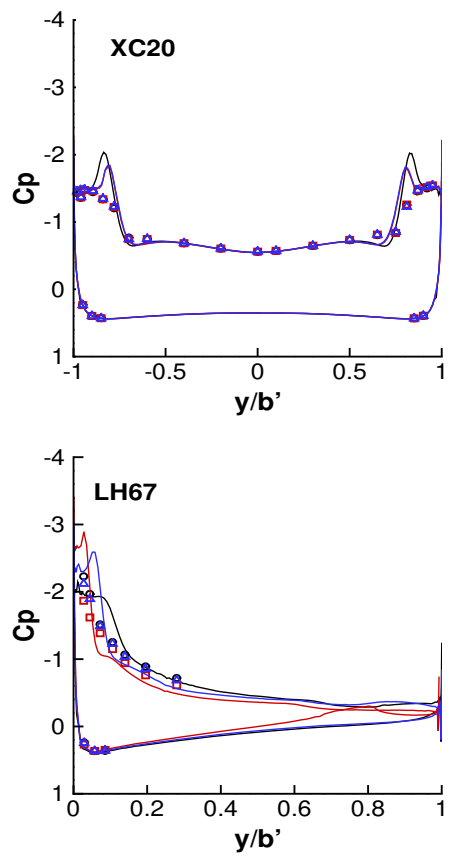

a) $\mathrm{AOA}=15^{\circ}$
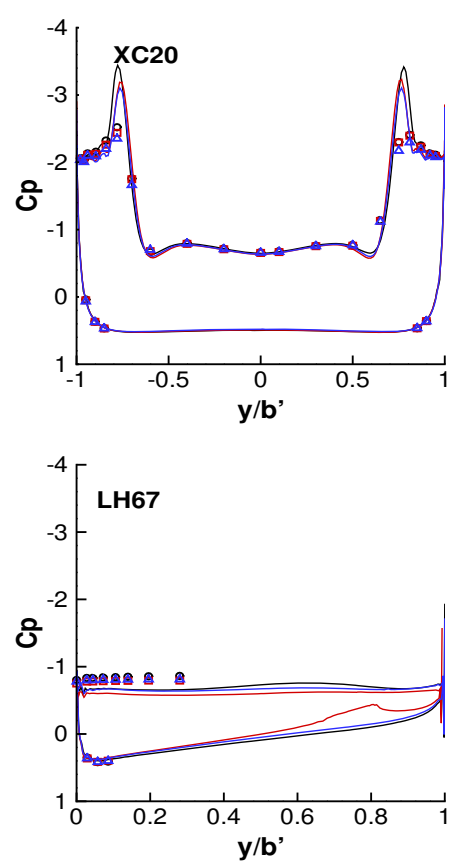
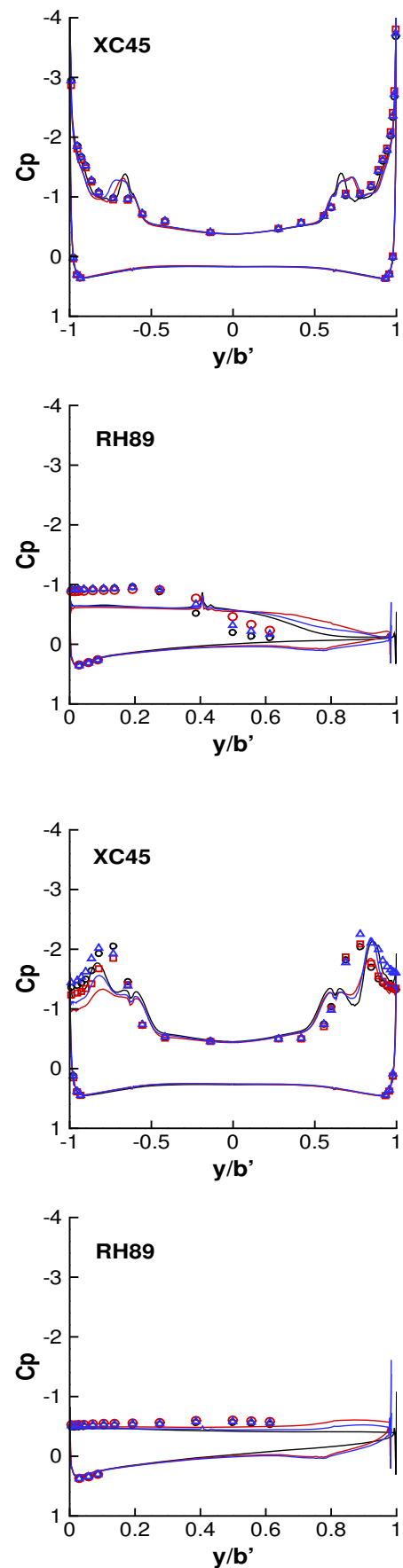
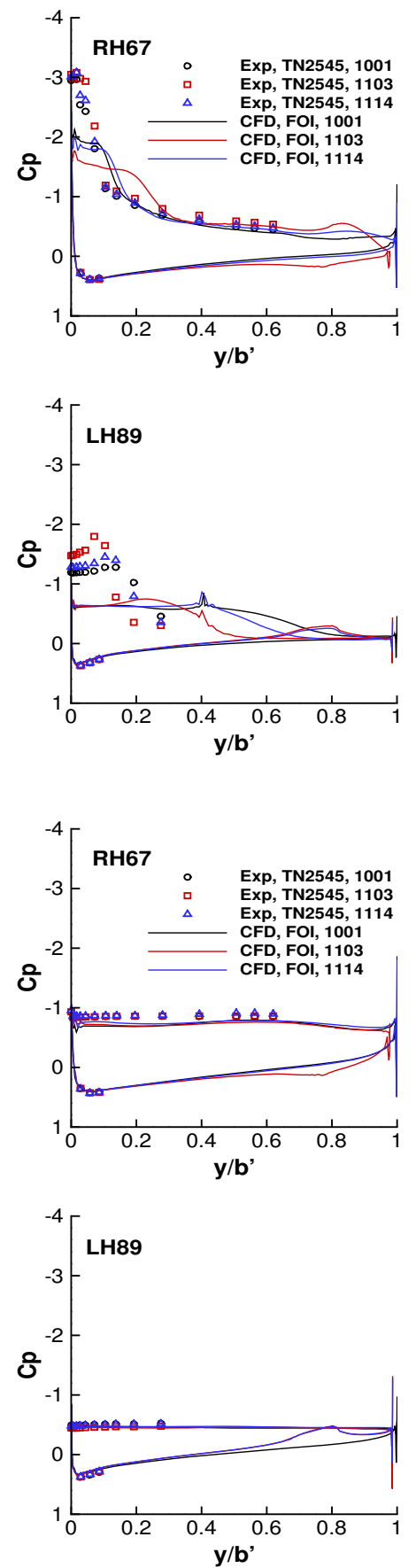

b) $\mathrm{AOA}=20^{\circ}$

Fig. 12 Cp sections comparing FOI time-accurate RANS and DLR experiments: $M_{\infty}=0.15, A O A=15$ and 20 deg, and $R e=1.6 \cdot 10^{6}$. 


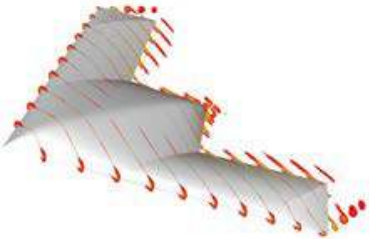

a) Base, $\alpha=0^{\circ}$

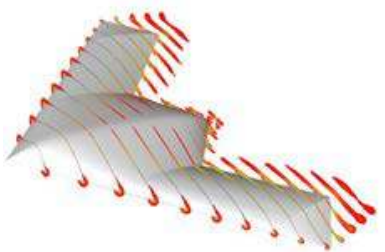

e) Base, $\alpha=5^{\circ}$

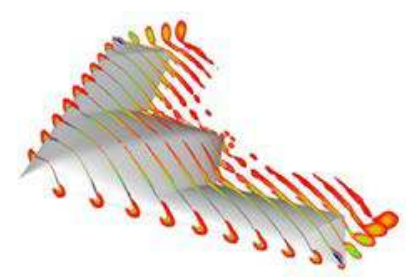

i) Base, $\alpha=10^{\circ}$

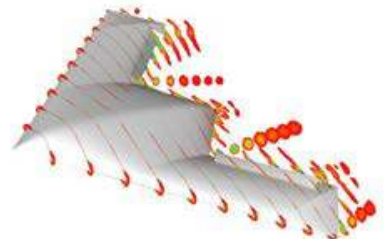

b) Case 1103, $\alpha=0^{\circ}$

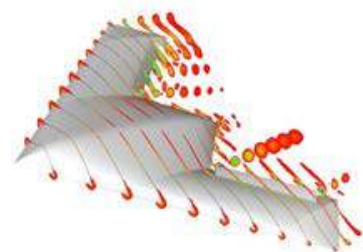

f) Case 1103, $\alpha=5^{\circ}$

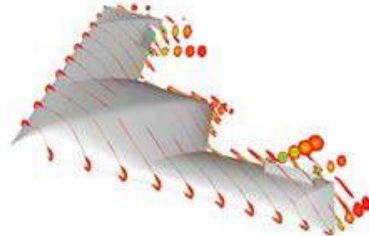

c) Case 1114, $\alpha=0^{\circ}$

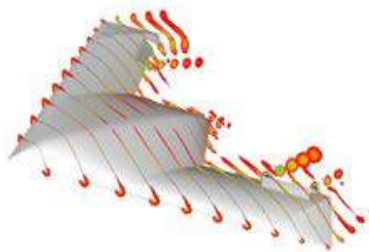

g) Case $1114, \alpha=5^{\circ}$

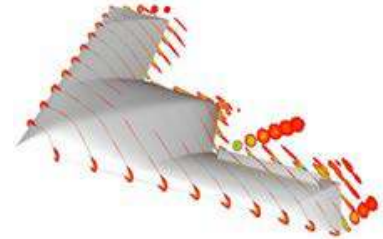

d) Case 1144, $\alpha=0^{\circ}$

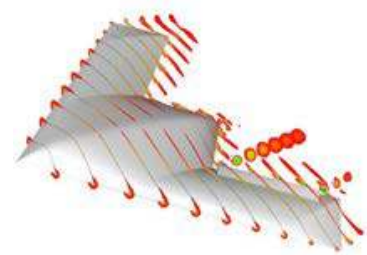

h) Case 1144, $\alpha=5^{\circ}$
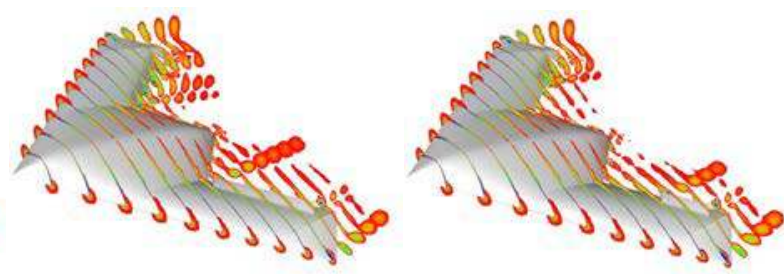

k) Case 1114, $\alpha=10^{\circ}$

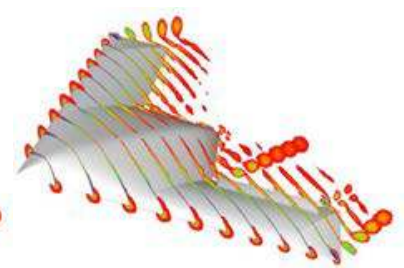

l) Case 1144, $\alpha=10^{\circ}$
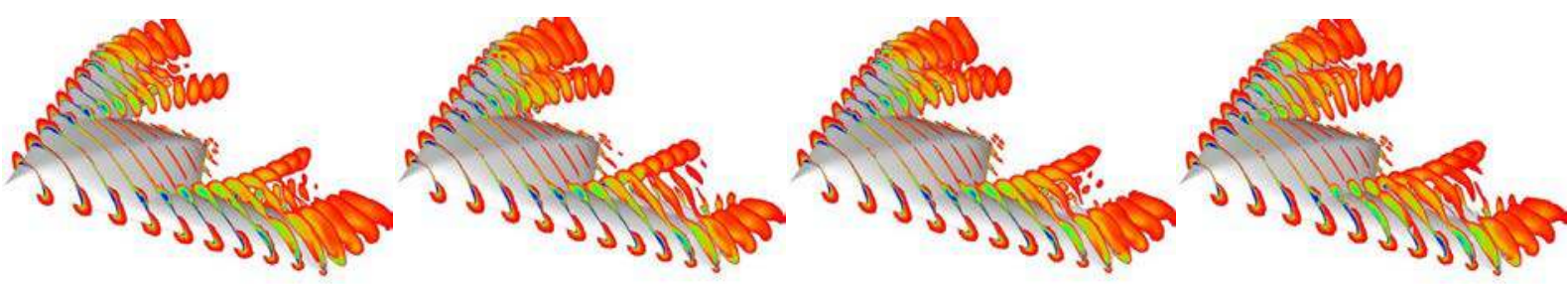

m) Base, $\alpha=15^{\circ}$

n) Case 1103, $\alpha=15^{\circ}$

o) Case 1114, $\alpha=15^{\circ}$

p) Case $1144, \alpha=15^{\circ}$

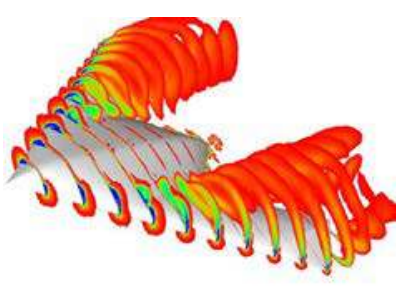

q) Base, $\alpha=20^{\circ}$
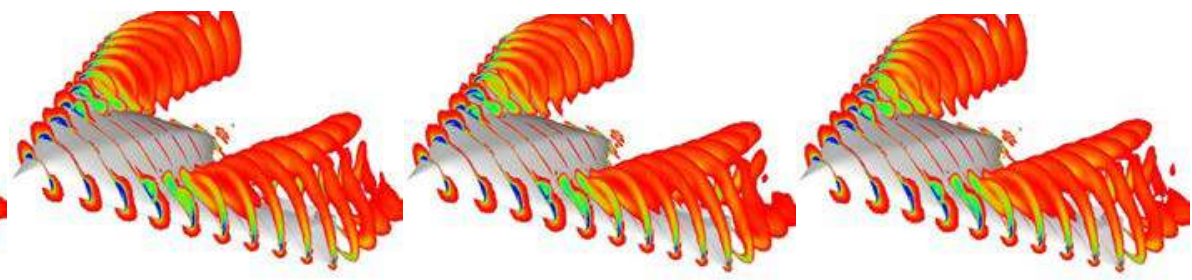

s) Case 1114, $\alpha=20^{\circ}$

t) Case $1144, \alpha=20^{\circ}$

r) Case 1103, $\alpha=20^{\circ}$

Vorticity

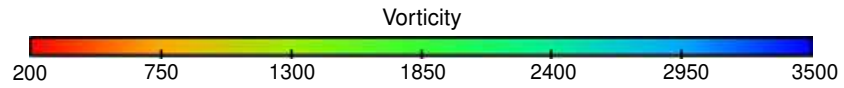

Fig. 13 FOI time-accurate RANS result showing vorticity on $x$-cut planes with $\Delta x=0.1 \mathrm{~m}, M_{\infty}=0.15$, and $R e=1.6 \cdot 10^{6}$.

\section{Evaluation of Leading-Edge Slats by ONERA}

As mentioned in the previous paragraph, the observed phenomenon of pitch down/up at a relatively low angle of attack limits the flight envelope. To improve the behavior of the SACCON design, mechanical devices have been designed, manufactured, and tested by ONERA. The goal of this flow control study is to manipulate the vortical structures in order to keep the tip vortex at high AOAs on the outboard wing as long as possible or to promote new vortical structures to balance the negative effects due to the displacement of the tip vortex from the rear part of the wing to the forepart of the wing. Several mechanical flow control devices are investigated. In this paper, and only the most efficient devices for pitchup improvement are presented. For a detailed description, readers may refer to the thesis by Morgand [18]. We consider here only two kinds of leading-edge slats (LESs), one short and one long, at two angular settings ( -15 and $-30 \mathrm{deg})$. The short one is located along the leading edge between $x / c_{R}=0.326$ and $x / c_{R}=0.46$. For the long slat, the location is between $x / c_{R}=0.326$ and $x / c_{R}=0.592$. The two LESs are upstream of the $\operatorname{MRP}\left(x / c_{R}=0.566\right)$.

Figure 21 shows the effectiveness of the two different LESs. The most significant effects are observed on the pitching moment coefficient. The long slats delay the pitch down/up and reduce the amplitude of the dip. The short slats infer more radical modifications: the phenomenon of pitch down is completely cancelled, but pitch up still exists. The effects on lift and drag are small (Fig. 21a). In addition, slats delay and reduce lateral asymmetries on the yaw coefficient (Fig. 21b). 


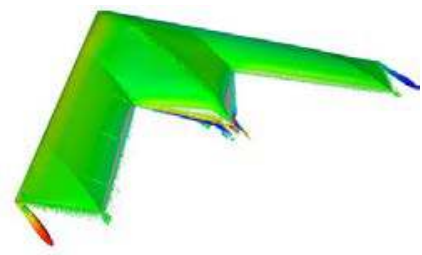

a) Base, $\alpha=0^{\circ}$

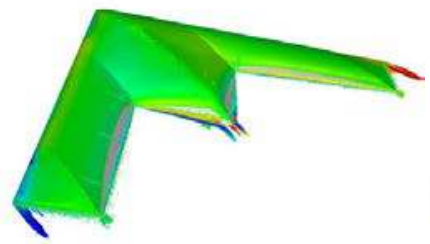

e) Base, $\alpha=5^{\circ}$

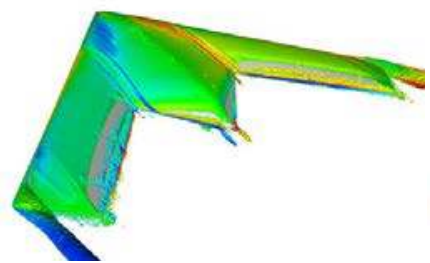

i) Base, $\alpha=10^{\circ}$

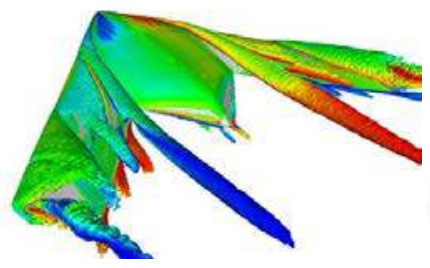

m) Base, $\alpha=15^{\circ}$

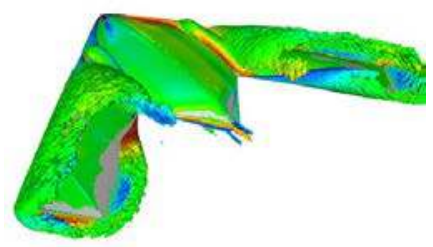

q) Base, $\alpha=20^{\circ}$

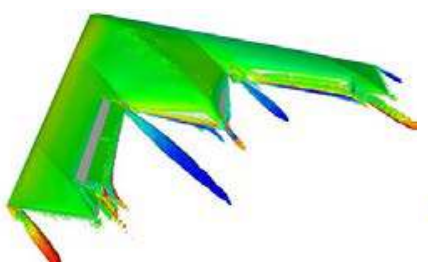

b) Case 1103, $\alpha=0^{\circ}$

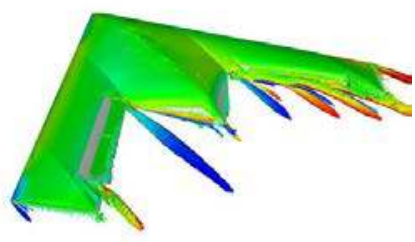

f) Case 1103, $\alpha=5^{\circ}$

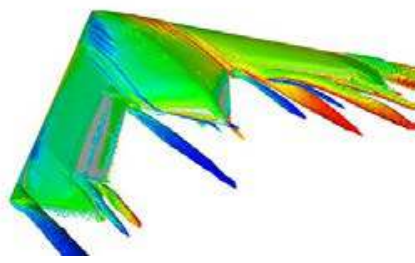

j) Case 1103, $\alpha=10^{\circ}$

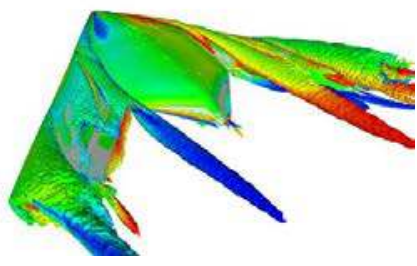

n) Case 1103, $\alpha=15^{\circ}$

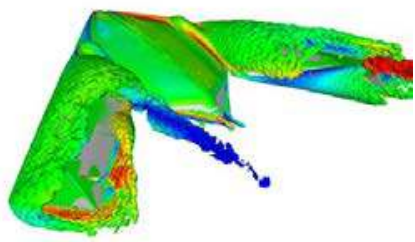

r) Case 1103, $\alpha=20^{\circ}$

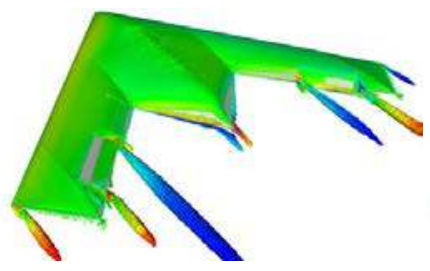

c) Case 1114, $\alpha=0^{\circ}$

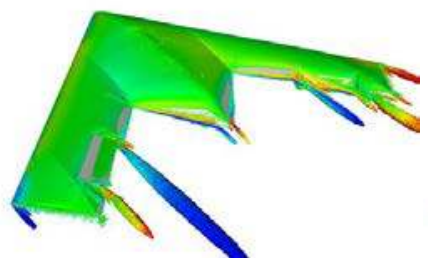

g) Case 1114, $\alpha=5^{\circ}$

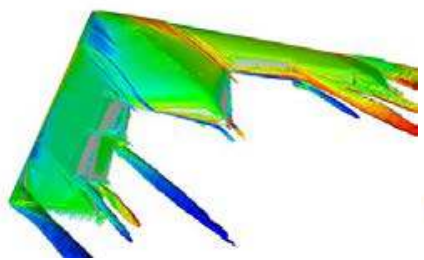

k) Case 1114, $\alpha=10^{\circ}$

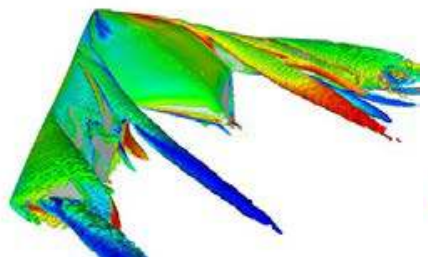

o) Case 1114, $\alpha=15^{\circ}$

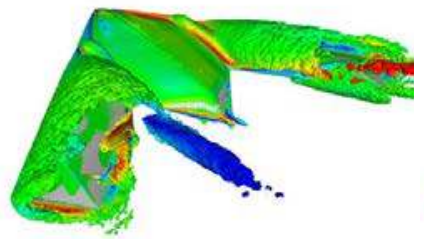

s) Case 1114, $\alpha=20^{\circ}$

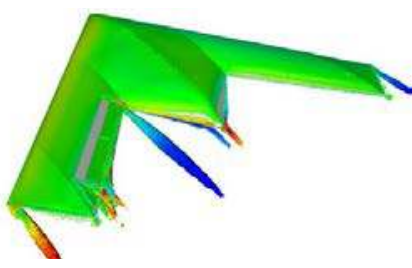

d) Case 1144, $\alpha=0^{\circ}$

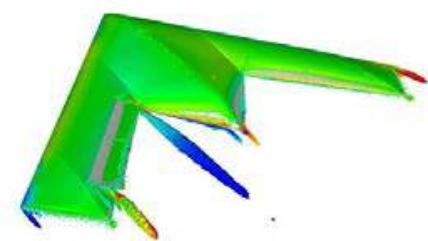

h) Case 1144, $\alpha=5^{\circ}$

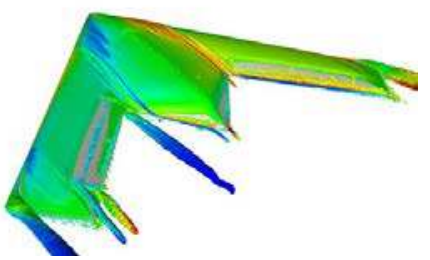

l) Case 1144, $\alpha=10^{\circ}$

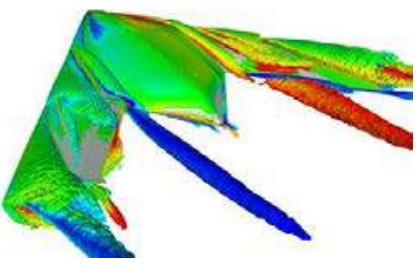

p) Case 1144, $\alpha=15^{\circ}$

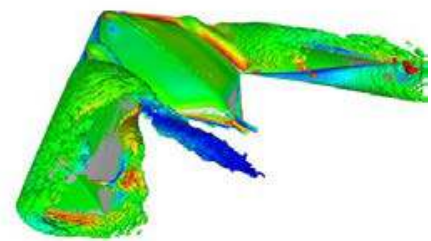

t) Case 1144, $\alpha=20^{\circ}$

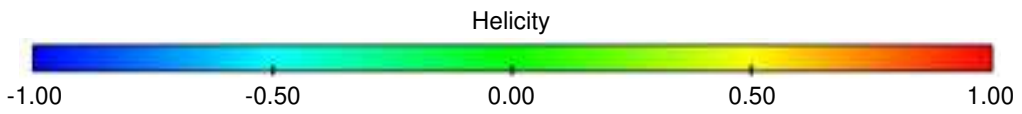

Fig. 14 FOI time-accurate RANS mean result showing isosurfaces of $Q$ criteria $(50,000)$ with relative helicity: $M_{\infty}=0.15$ and $R e=1.6 \cdot 10^{6}$.

The efficiency of the mechanical LES devices was also assessed through numerical simulation by ONERA. Before the experimental campaign, to support the design, a parametric study of LESs was made on the shape, position, width, and deflection. After the tests, numerical simulations were carried out for validation and for in-depth flowfield investigation.

Numerical simulations of the short slat show a large effect on the pitching moment coefficient (Fig. 22) by reducing the nonlinearity. There is still a dip at $\mathrm{AOA}=17 \mathrm{deg}$ in the numerical data for the direct comparison with the $10-\mathrm{mm}$-wide slat. The dip is removed by simulating the wider $20 \mathrm{~mm}$ slat. If the angle of the $20 \mathrm{~mm}$ slat is increased to $-30 \mathrm{deg}$, the pitching moment drops somewhat again. The triangle short slat shows an efficiency more or less comparable to that of the wider short slat. The efficiency of the slats measured through wind-tunnel tests are therefore confirmed but for larger widths.

Figure 23 shows the pressure coefficient on the upper surface with and without the control device (short slat at $x / c_{R}=0.326$ ) at $\mathrm{AOA}=17 \mathrm{deg}$. The surface pressure distribution exhibits the suction caused by the vortices. Figure 23 a shows the location and the development of the apex vortex (bottom triangles) and tip vortex (top triangles) without the device. The onset of tip vortex occurs at the leading edge upstream of the $\operatorname{MRP}\left(x / c_{R}=0.566\right)$.

The influence of the LES (short rectangular plate, width $20 \mathrm{~mm}$ ) on the flow topology can be observed in Fig. 23b. The apex vortex still exists, but a new vortex is located at the upstream position of the LES 


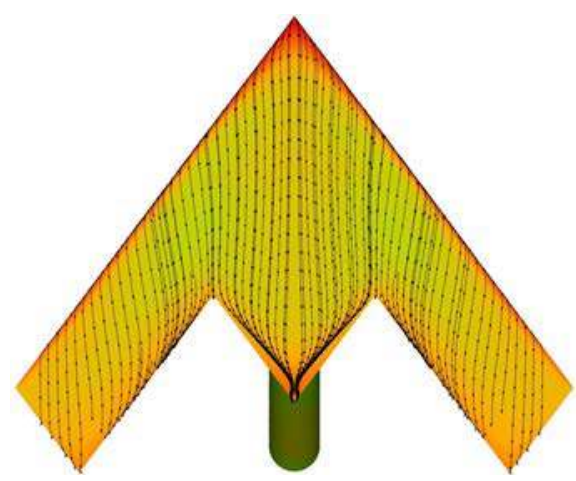

a) Base, $\alpha=0^{\circ}$

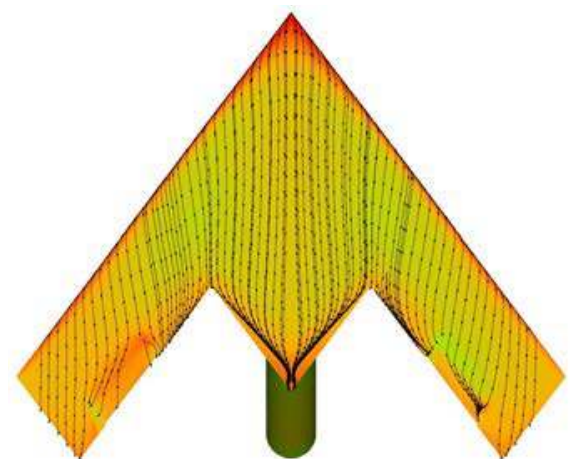

c) Case 1114, $\alpha=0^{\circ}$

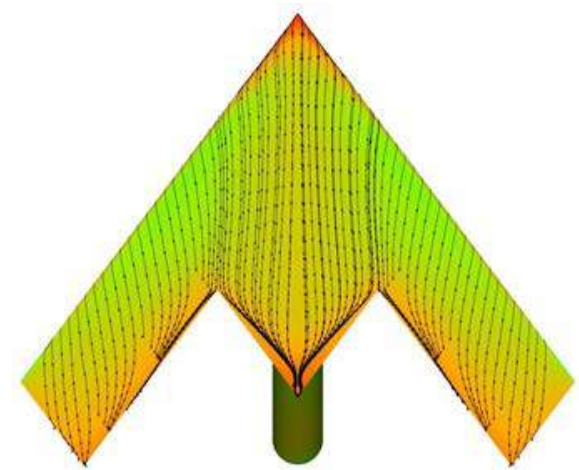

e) Base, $\alpha=5^{\circ}$

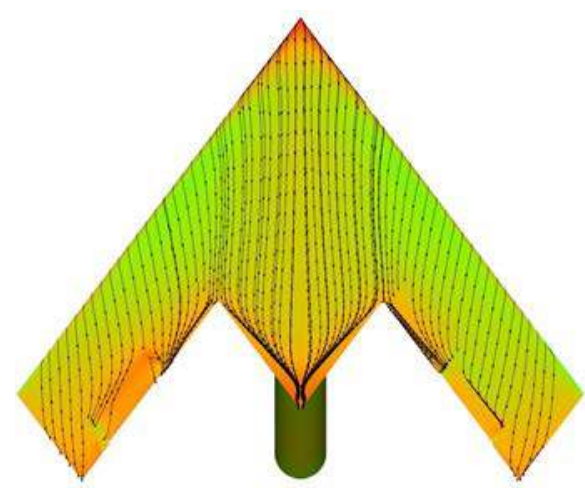

g) Case 1114, $\alpha=5^{\circ}$

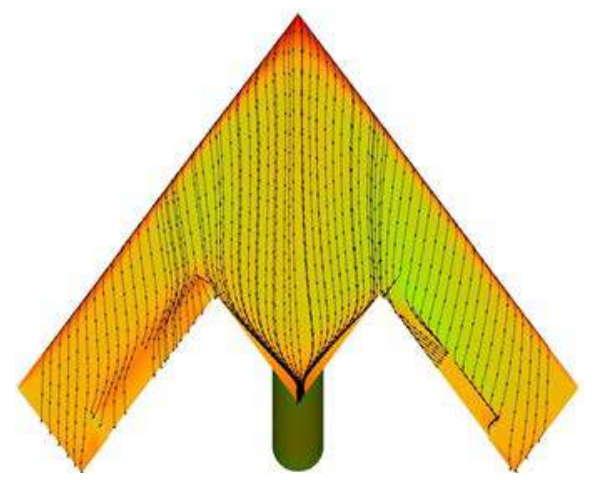

b) Case 1103, $\alpha=0^{\circ}$

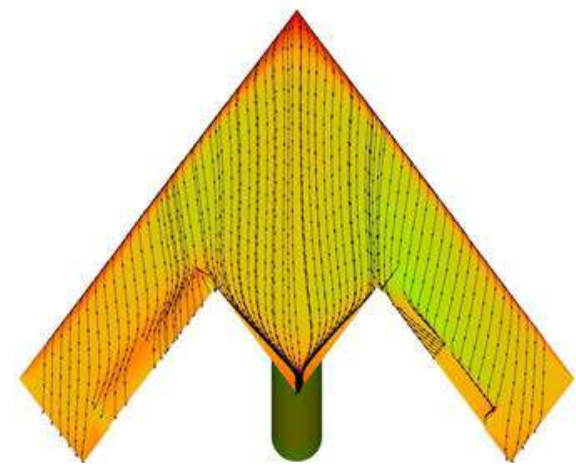

d) Case 1144, $\alpha=0^{\circ}$

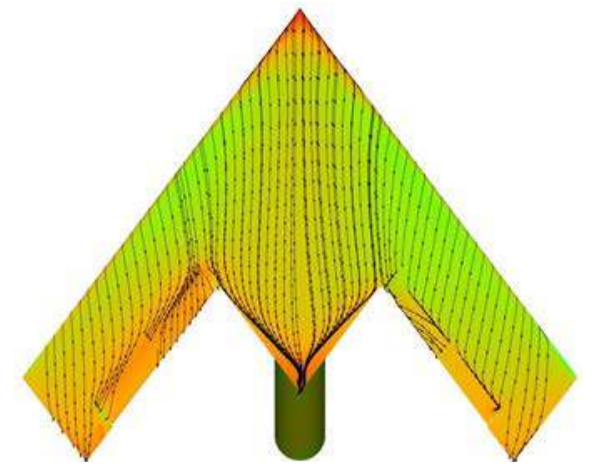

f) Case 1103, $\alpha=5^{\circ}$

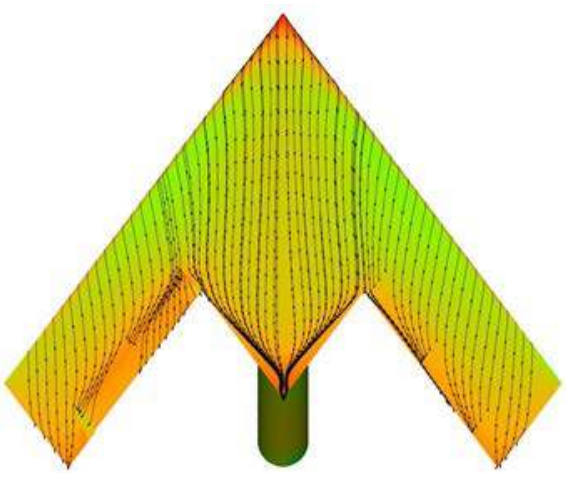

h) Case 1144, $\alpha=5^{\circ}$

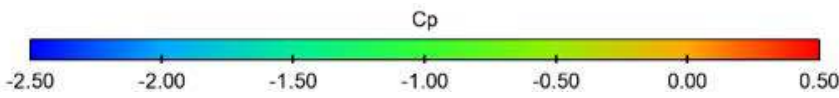

Fig. 15 FOI time-accurate RANS result showing upper surface pressure and skin-friction lines: $M_{\infty}=0.15, \mathrm{AOA}=0$ and $5 \mathrm{deg}$, and $\operatorname{Re}=1.6 \cdot 10^{6}$ (sting included in these pictures). 


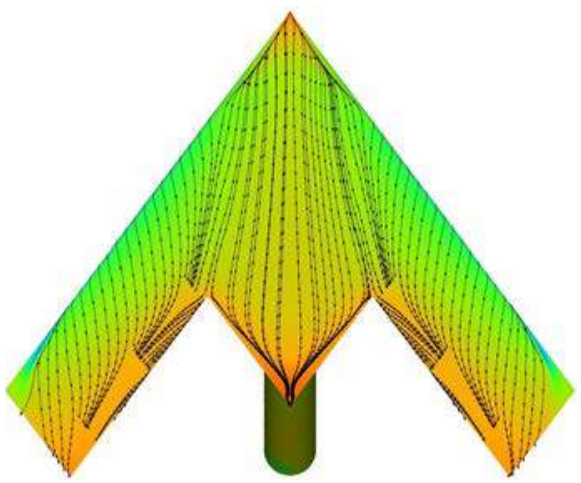

a) Base, $\alpha=10^{\circ}$

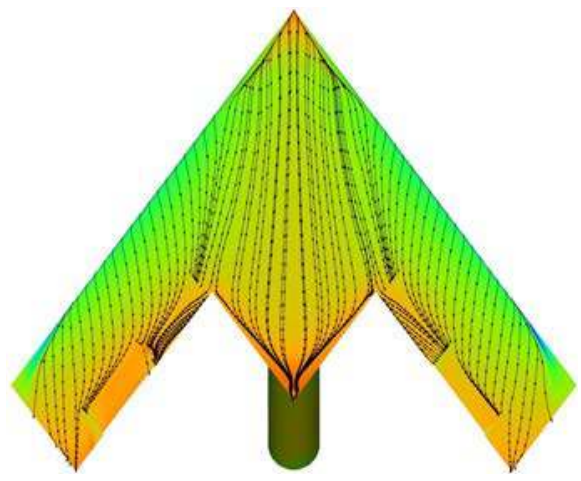

c) Case 1114, $\alpha=10^{\circ}$

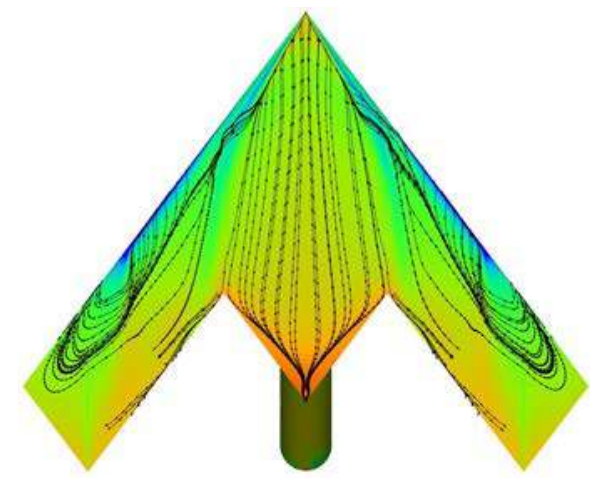

e) Base, $\alpha=15^{\circ}$

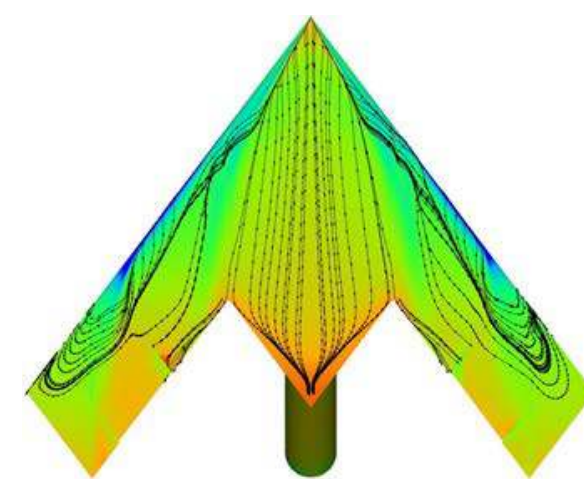

g) Case 1114, $\alpha=15^{\circ}$

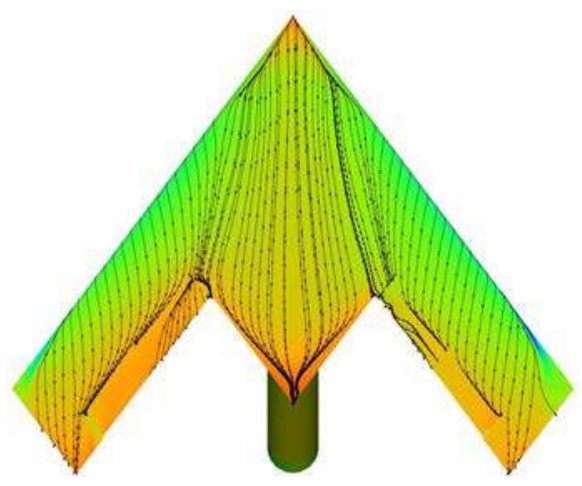

b) Case 1103, $\alpha=10^{\circ}$

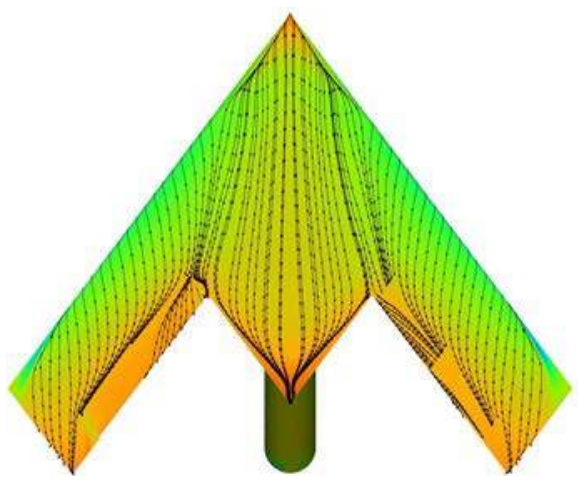

d) Case 1144, $\alpha=10^{\circ}$

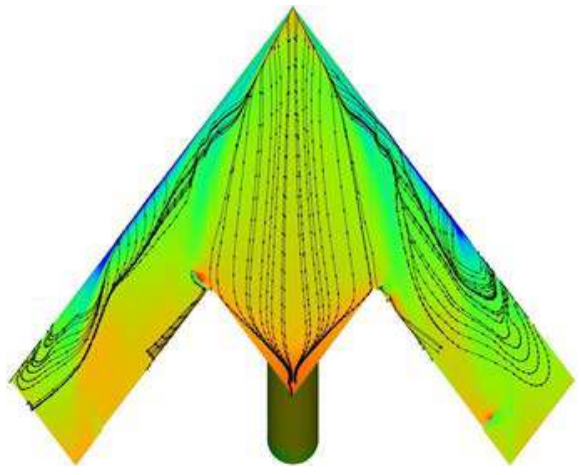

f) Case 1103, $\alpha=15^{\circ}$

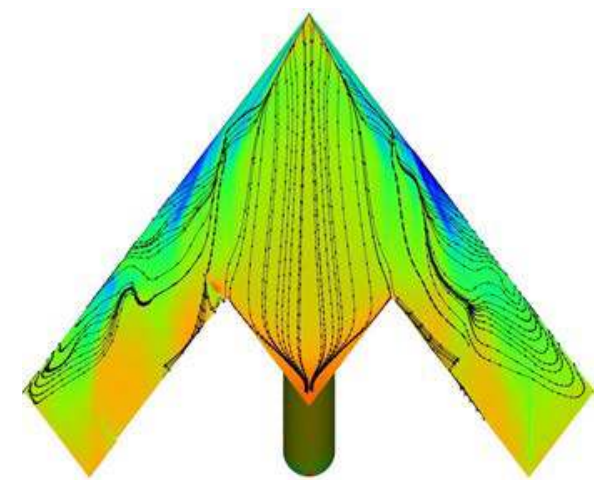

h) Case 1144, $\alpha=15^{\circ}$

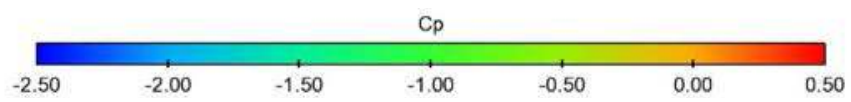

Fig. 16 FOI time-accurate RANS result showing upper surface pressure and skin-friction lines: $M_{\infty}=0.15, \mathrm{AOA}=10$ and $5 \mathrm{deg}$, and $R e=1.6 \cdot 10^{6}$ (sting included in these pictures). 


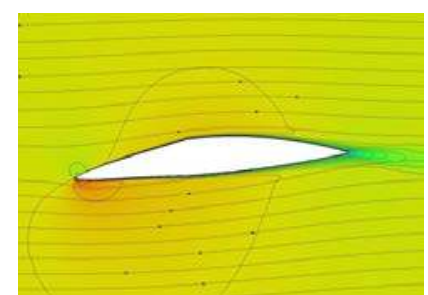

a) Base, $\alpha=0^{\circ}$

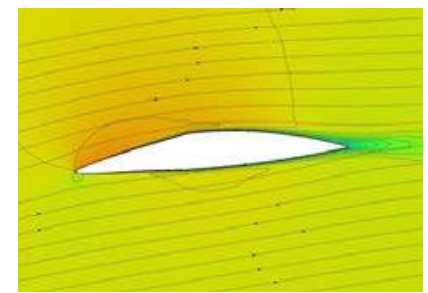

e) Base, $\alpha=5^{\circ}$

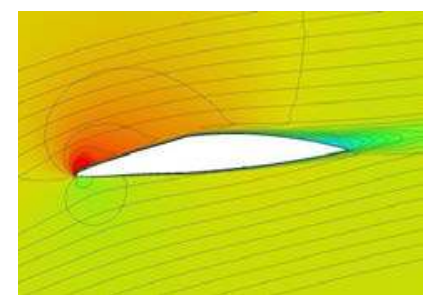

i) Base, $\alpha=10^{\circ}$

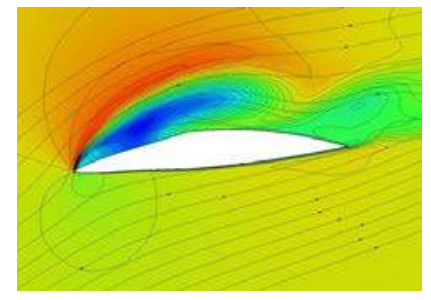

m) Base, $\alpha=15^{\circ}$

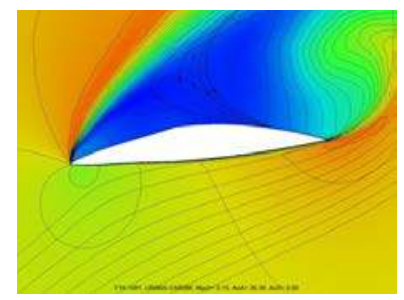

q) Base, $\alpha=20^{\circ}$

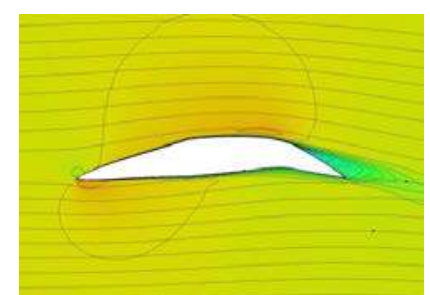

b) Case 1103, $\alpha=0^{\circ}$

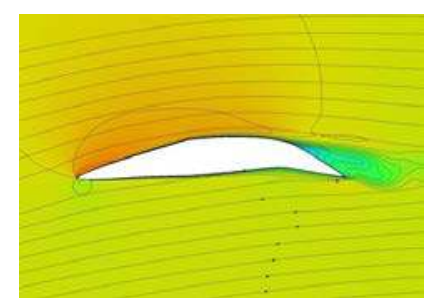

f) Case 1103, $\alpha=5^{\circ}$

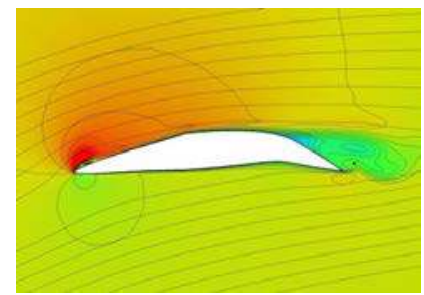

j) Case 1103, $\alpha=10^{\circ}$

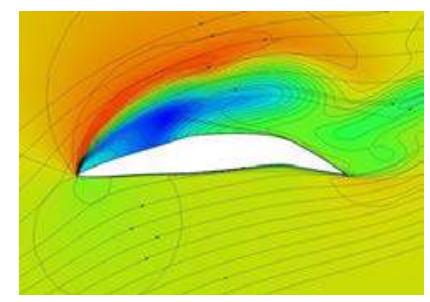

n) Case 1103, $\alpha=15^{\circ}$

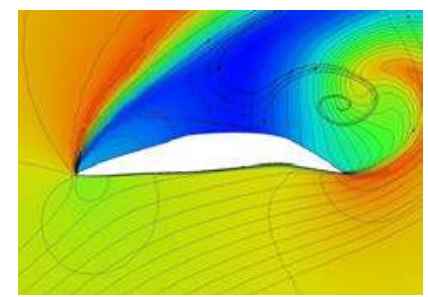

r) Case 1103, $\alpha=20^{\circ}$

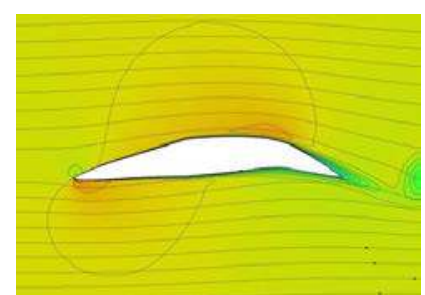

c) Case 1114, $\alpha=0^{\circ}$

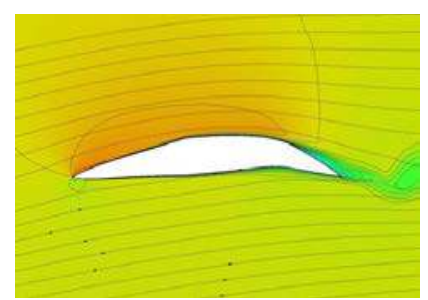

g) Case 1114, $\alpha=5^{\circ}$

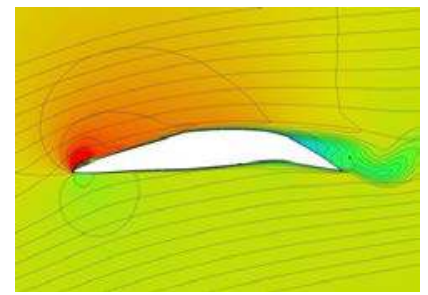

k) Case 1114, $\alpha=10^{\circ}$

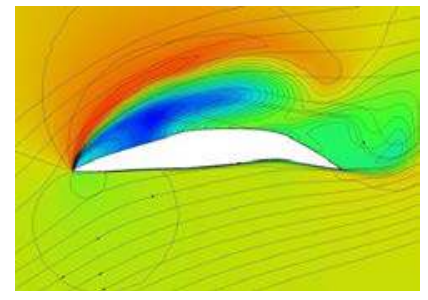

o) Case 1114, $\alpha=15^{\circ}$

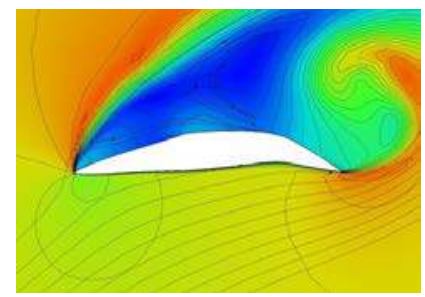

s) Case 1114, $\alpha=20^{\circ}$

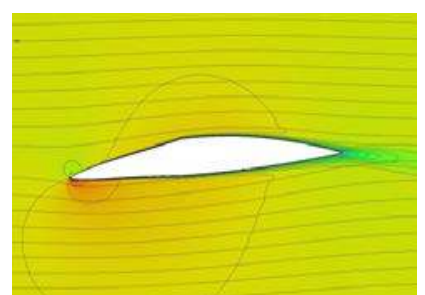

d) Case 1144, $\alpha=0^{\circ}$

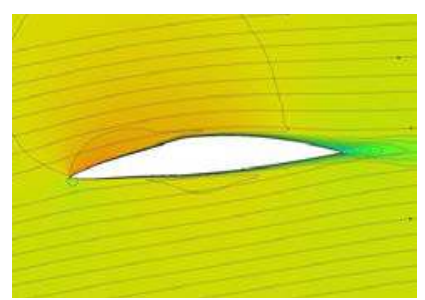

h) Case 1144, $\alpha=5^{\circ}$

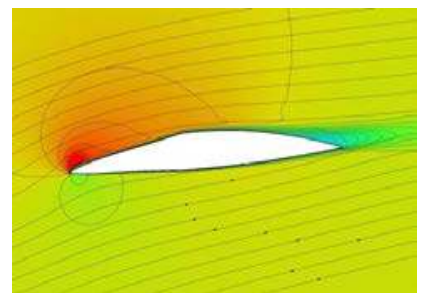

I) Case 1144, $\alpha=10^{\circ}$

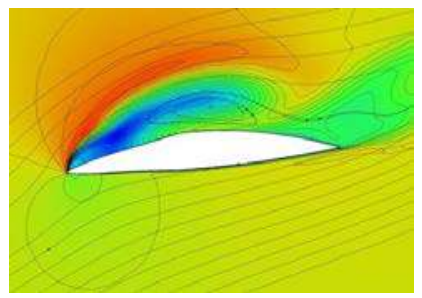

p) Case 1144, $\alpha=15^{\circ}$

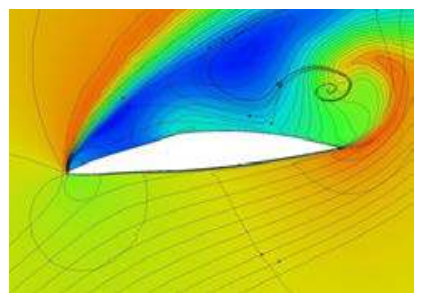

t) Case 1144, $\alpha=20^{\circ}$

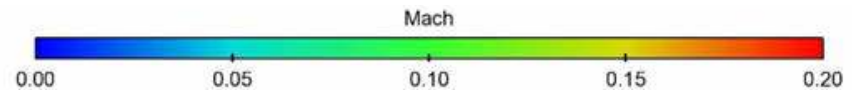

Fig. 17 FOI RANS (TA mean) result of Mach and streamlines at outer right control (cut at RH89): $M_{\infty}=0.15$ and $R e=1.6 \cdot 10^{6}$.

and a third vortex develops in the rear part of the wing. This third vortex depends on the shape of the device and its spanwise position [18]. The LES generates a new vortex system that promotes suction close to the leading edge and moves the tip vortex away from the inner rear wing, and therefore increases the pitching moment at a 17 deg AOA.

Comparison of the dimensionless axial velocity at $\mathrm{AOA}=$ 17 deg with and without LESs for two cross sections is presented in Fig. $24\left(x / c_{R}=0.55\right.$ and $\left.x / c_{R}=0.77\right)$. The distribution of the pressure coefficient $\mathrm{Cp}$ is plotted on the same figures (thick line) as a function of the local span. At $x / c_{R}=0.55$ (Figs. 24a and 24c), the dimensionless axial velocity distribution exhibits the presence of two co-rotating vortices. Without slats, these vortices correspond to the apex and tip vortices. The presence of the slat has two major effects on these vortices: the apex vortex is intensified, and the second one is located closer to leading edge; this vortex is certainly directly induced by the LES. The locations of the vortices are confirmed by the lowpressure peaks; this also shows that the tip vortex dominates the flowfield. At $x / c_{R}=0.77$ (Fig. 24b), without LESs, the two corotating vortices as described before still exist, but the apex vortex is close to the trailing edge and the tip vortex is located at midchord. With LESs, a new vortex can be seen; three vortices therefore develop 


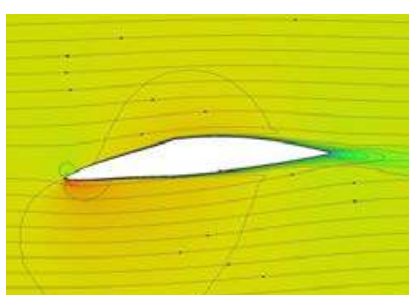

a) Base, $\alpha=0^{\circ}$

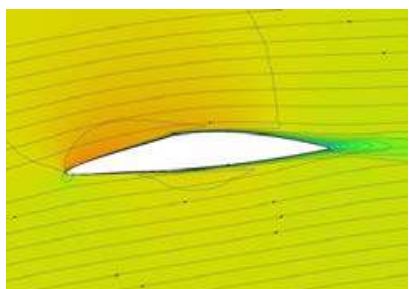

e) Base, $\alpha=5^{\circ}$

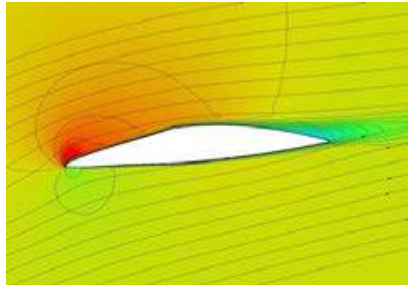

i) Base, $\alpha=10^{\circ}$

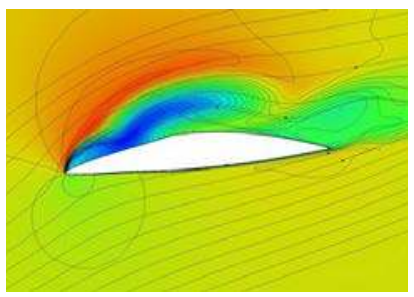

m) Base, $\alpha=15^{\circ}$

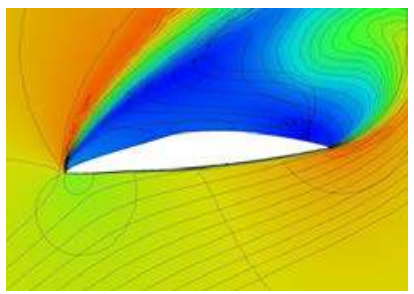

q) Base, $\alpha=20^{\circ}$

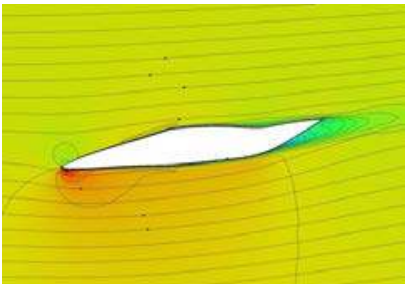

b) Case 1103, $\alpha=0^{\circ}$

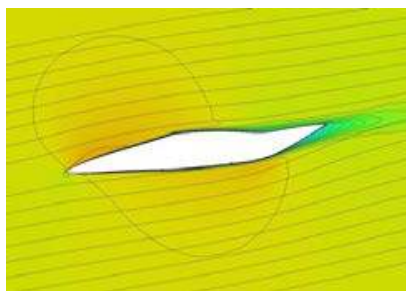

f) Case 1103, $\alpha=5^{\circ}$

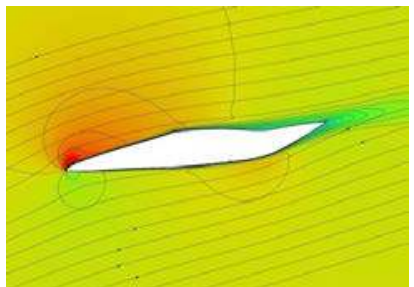

j) Case 1103, $\alpha=10^{\circ}$

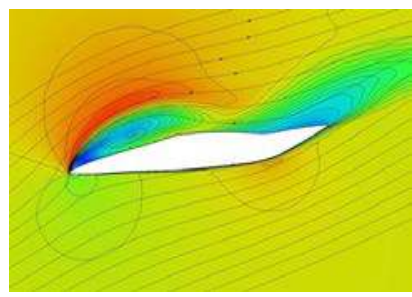

n) Case 1103, $\alpha=15^{\circ}$

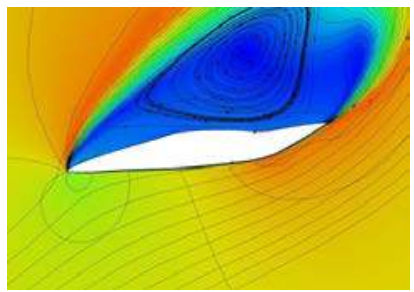

r) Case 1103, $\alpha=20^{\circ}$

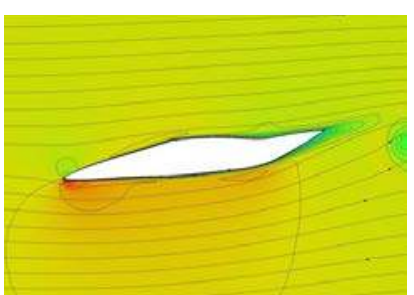

c) Case 1114, $\alpha=0^{\circ}$

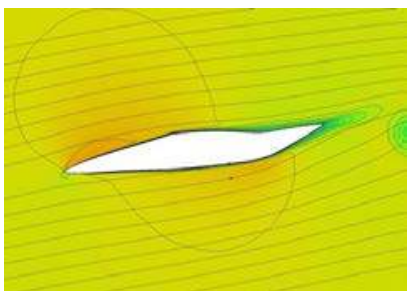

g) Case 1114, $\alpha=5^{\circ}$

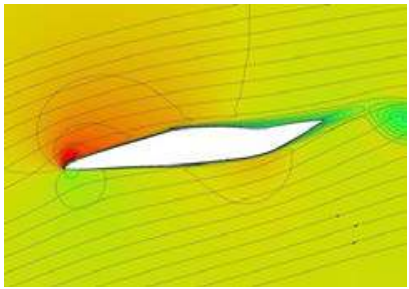

k) Case 1114, $\alpha=10^{\circ}$

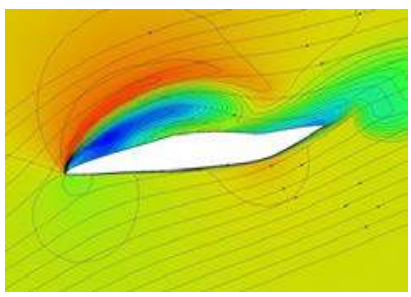

o) Case 1114, $\alpha=15^{\circ}$

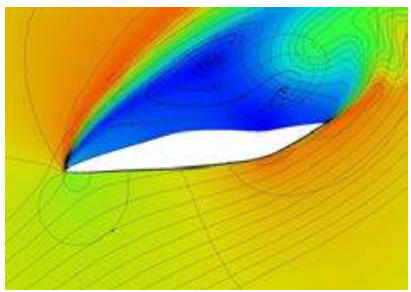

s) Case 1114, $\alpha=20^{\circ}$

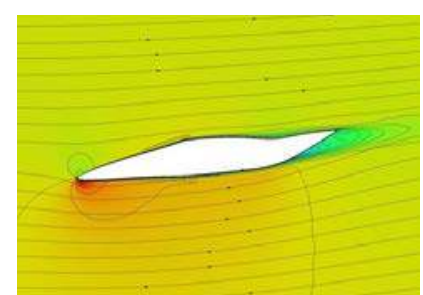

d) Case 1144, $\alpha=0^{\circ}$

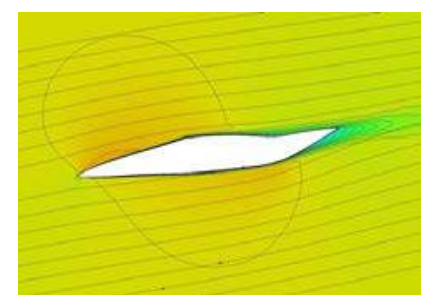

h) Case 1144, $\alpha=5^{\circ}$

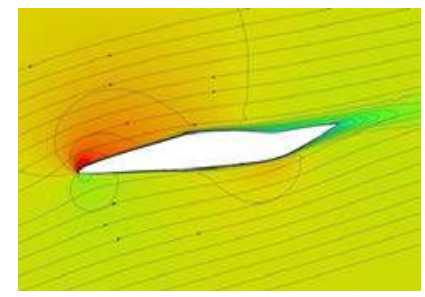

I) Case 1144, $\alpha=10^{\circ}$

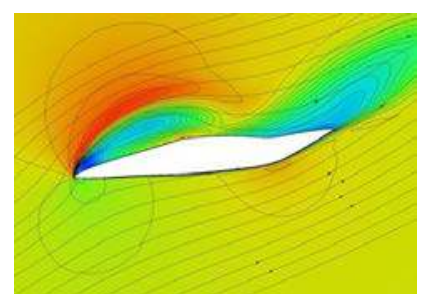

p) Case 1144, $\alpha=15^{\circ}$

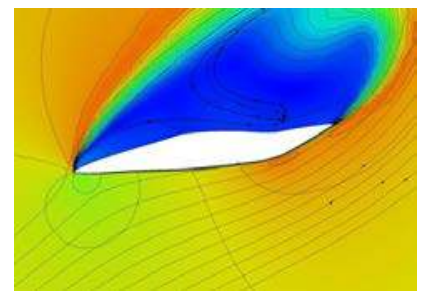

t) Case 1144, $\alpha=20^{\circ}$

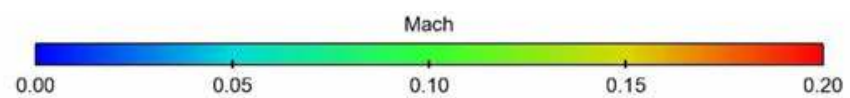

Fig. 18 FOI RANS (TA mean) result of Mach and streamlines at outer left control $\left(\operatorname{cut}\right.$ at $\left.\mathrm{LH}^{\mathrm{H} 9}\right): \mathrm{M}_{\infty}=0.15$ and $R e=1.6 \cdot 10^{6}$.

on the upper surface. The values of the axial velocity in the apex and slat vortices reach about $0.2 U_{\infty}$; this low value indicates that the sudden vortex breakdown may appear [28,30]. The combination of a high suction peak and high axial velocity close to the leading edge indicates that the cross section is close to the onset of the third vortex.

At $\mathrm{AOA}=17 \mathrm{deg}$ without LESs, the pitchdown effect is maximum, as shown in Fig. 22. This is explained by large areas of low pressure in the aft part of the wing. With the LESs, the new vortex system promotes a pitchup moment due to the reduction of the level of low pressure in a region situated aft of the MRP and additional vortical loads on the middle of the wing $\left(x / c_{R}=0.36\right.$ and $\left.x / c_{R}=0.56\right)$.

\section{Estimation of Dynamic Derivatives by ONERA}

Dynamic derivatives have also been evaluated by ONERA. The simulations of pitching motions are presented and compared to the available experimental data for the clean configuration. The boundary of the body is forced to oscillate with a simple sinusoidal function, $\Theta(t)=\Delta \Theta \sin (\omega t)$ applied about the $y$ axis without changing the 


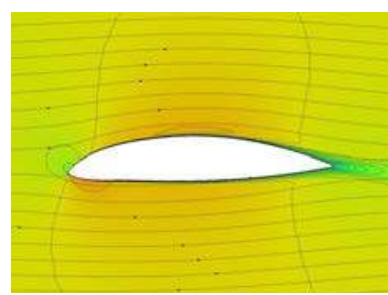

a) Base, $\alpha=0^{\circ}$

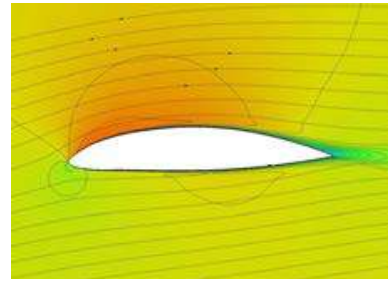

e) Base, $\alpha=5^{\circ}$

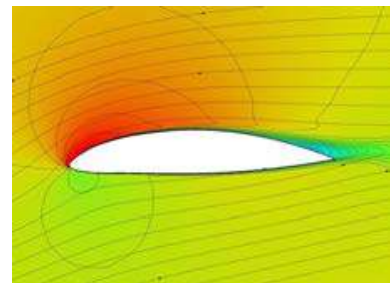

i) Base, $\alpha=10^{\circ}$

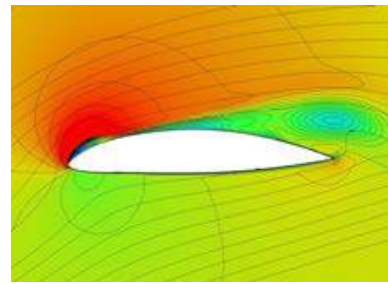

m) Base, $\alpha=15^{\circ}$

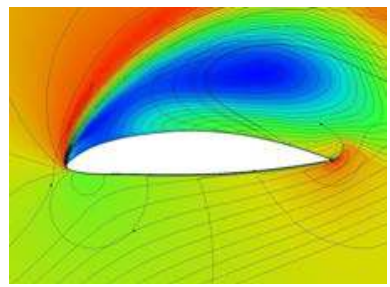

q) Base, $\alpha=20^{\circ}$

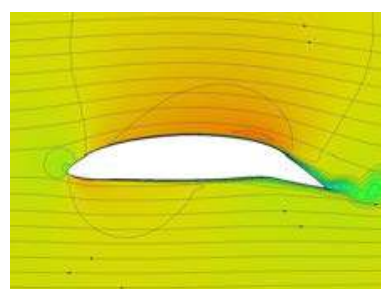

b) Case 1103, $\alpha=0^{\circ}$

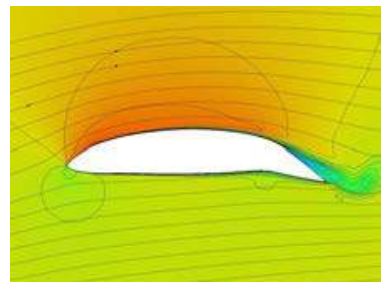

f) Case 1103, $\alpha=5^{\circ}$

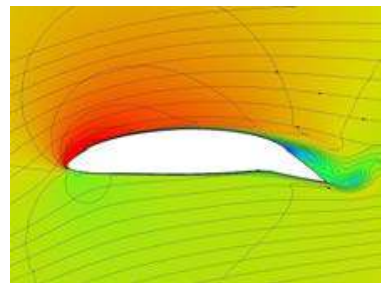

j) Case 1103, $\alpha=10^{\circ}$

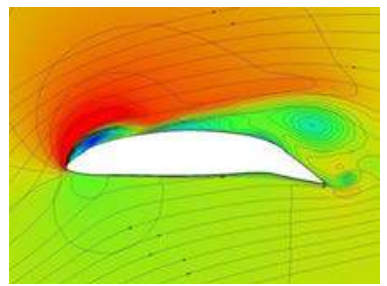

n) Case 1103, $\alpha=15^{\circ}$

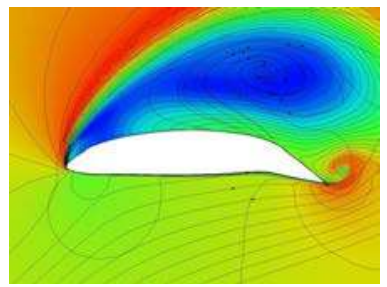

r) Case 1103, $\alpha=20^{\circ}$

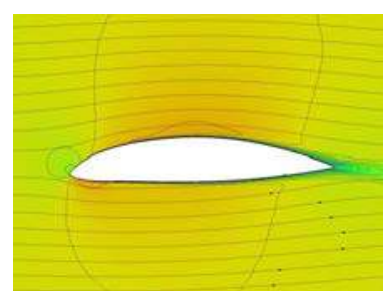

c) Case 1114, $\alpha=0^{\circ}$

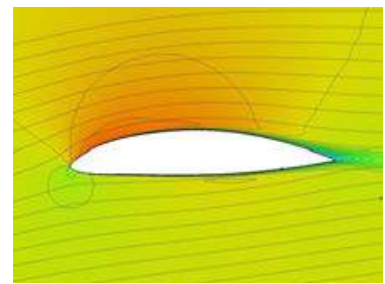

g) Case 1114, $\alpha=5^{\circ}$

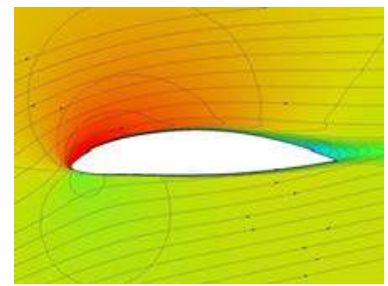

k) Case 1114, $\alpha=10^{\circ}$

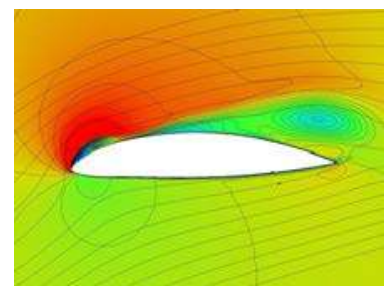

o) Case 1114, $\alpha=15^{\circ}$

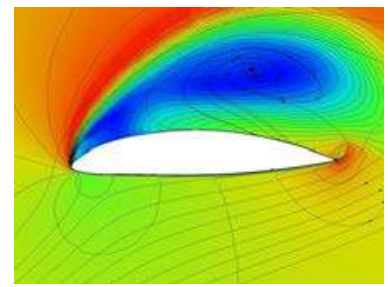

s) Case 1114, $\alpha=20^{\circ}$

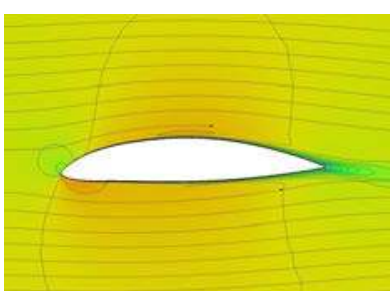

d) Case 1144, $\alpha=0^{\circ}$

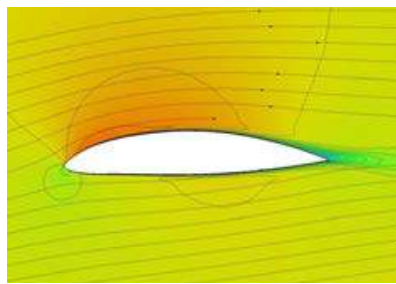

h) Case 1144, $\alpha=5^{\circ}$

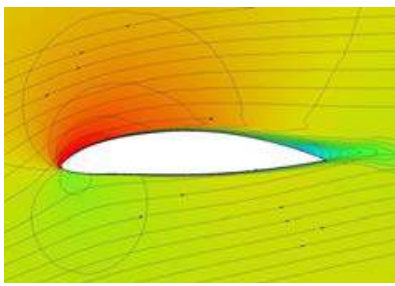

I) Case 1144, $\alpha=10^{\circ}$

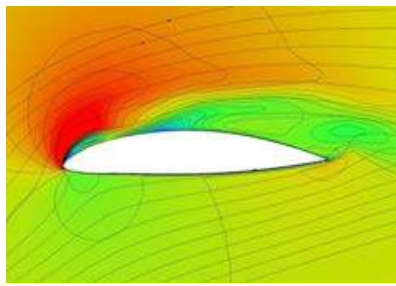

p) Case 1144, $\alpha=15^{\circ}$

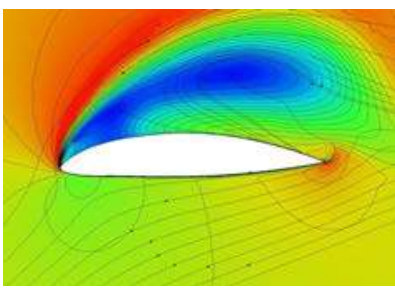

t) Case 1144, $\alpha=20^{\circ}$

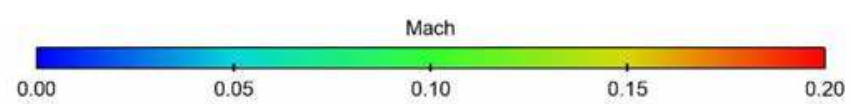

Fig. 19 FOI RANS (TA mean) result of Mach and streamlines at inner right control (cut at RH67): $M_{\infty}=0.15$ and $R e=1.6 \cdot 10^{6}$.

incidence of the flow. This coupling induces a pure effect of pitch rate. The unsteady simulations have been performed with the coarser grid (mesh 1) in order to save some computational time. Several AOAs have been computed to cover a large part of the aerodynamic range as in the experiments. The amplitude of oscillations is $5 \mathrm{deg}$, and the frequency is $1 \mathrm{~Hz}$. The simulations use 400 time steps per complete cycle $\left(\Delta t=2.510^{-3} \mathrm{~s}\right)$ to reproduce the sinusoidal motion. After an initial transient of $1 / 4$ cycle, the solution is periodic. The effects of the $q$ solicitation are measured when the model passes through the initial value during the up- and downstrokes at 200 and $400 \Delta t$. Results are presented in the a later paragraph.
The effects of moving the center of rotation backward on the $q$ derivatives are evaluated by both dynamic tests in the wind tunnel and calculations.

Complementary tests have been done on a rotary balance [31] with an additional mechanical part in order to move backward the center of rotation from the initial location at $x / c_{R}=63.5 \%$ to $x / c_{R}=80.9 \%$. We focus on the effects of the pitch rate on the normal force and pitching moment coefficients, and comparisons are made between identifications from the coning tests on a rotary balance at $\mathrm{AOA}=0$, 5,10 , and $15 \mathrm{deg}$ and a sideslip of $10 \mathrm{deg}$ for the two locations of the center of rotation. 


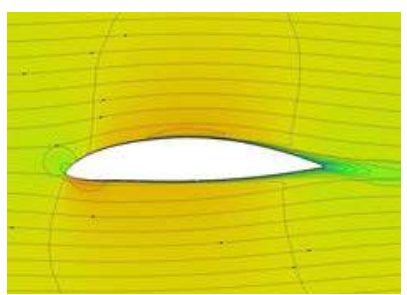

a) Base, $\alpha=0^{\circ}$

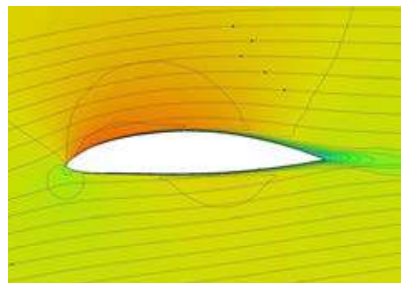

e) Base, $\alpha=5^{\circ}$

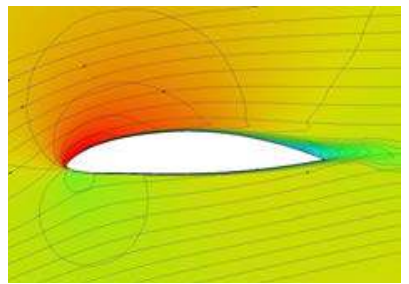

i) Base, $\alpha=10^{\circ}$

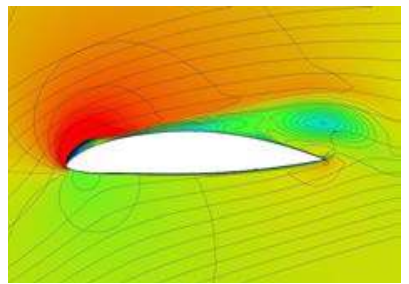

m) Base, $\alpha=15^{\circ}$

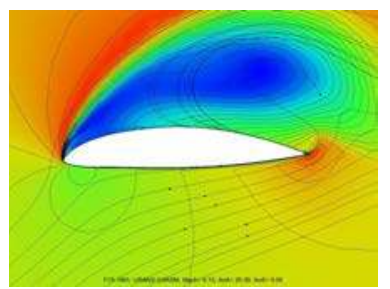

q) Base, $\alpha=20^{\circ}$

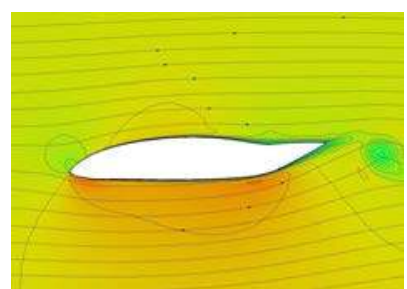

b) Case 1103, $\alpha=0^{\circ}$

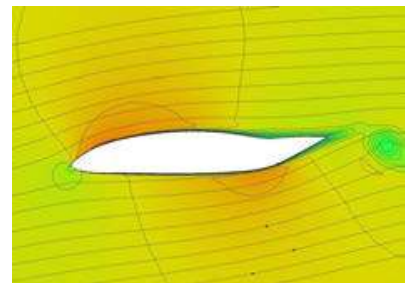

f) Case 1103, $\alpha=5^{\circ}$

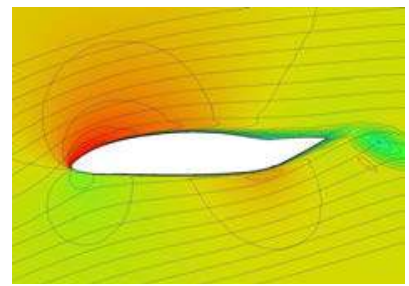

j) Case 1103, $\alpha=10^{\circ}$

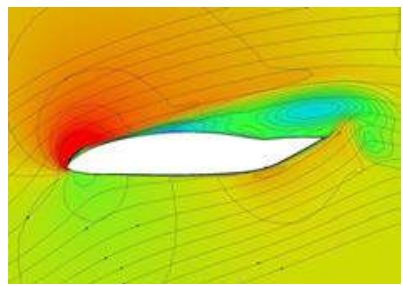

n) Case 1103, $\alpha=15^{\circ}$

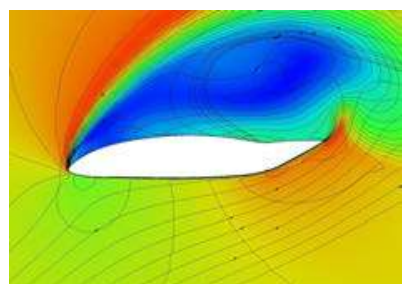

r) Case 1103, $\alpha=20^{\circ}$

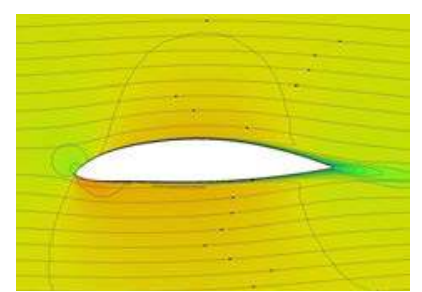

c) Case 1114, $\alpha=0^{\circ}$

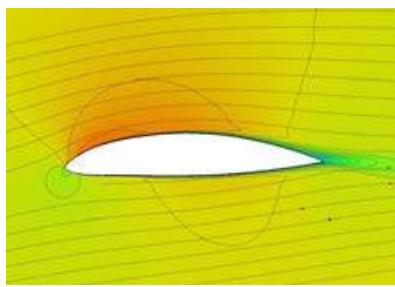

g) Case 1114, $\alpha=5^{\circ}$

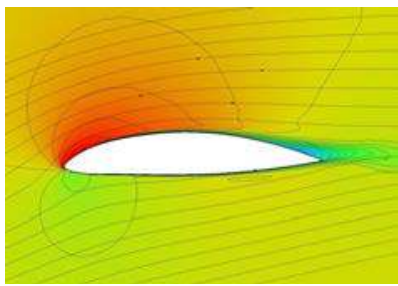

k) Case 1114, $\alpha=10^{\circ}$

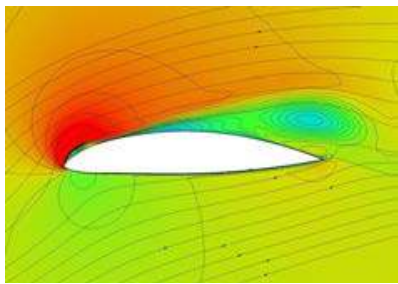

o) Case 1114, $\alpha=15^{\circ}$

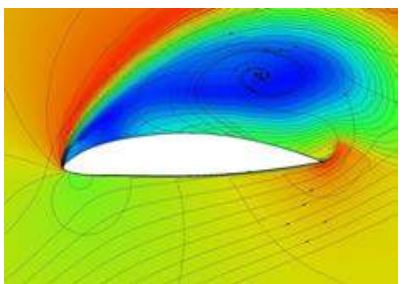

s) Case 1114, $\alpha=20^{\circ}$

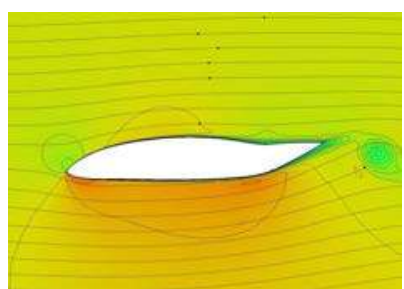

d) Case 1144, $\alpha=0^{\circ}$

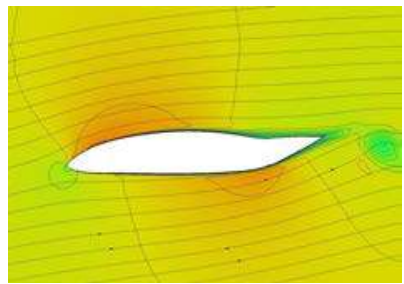

h) Case 1144, $\alpha=5^{\circ}$

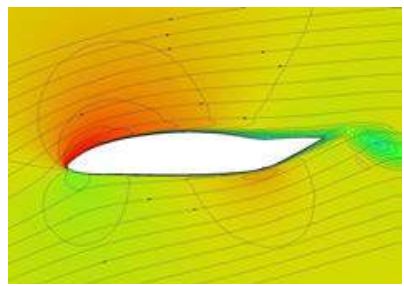

l) Case 1144, $\alpha=10^{\circ}$

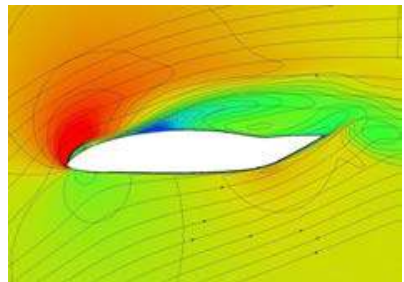

p) Case 1144, $\alpha=15^{\circ}$

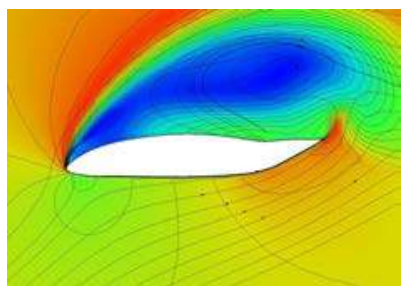

t) Case 1144, $\alpha=20^{\circ}$

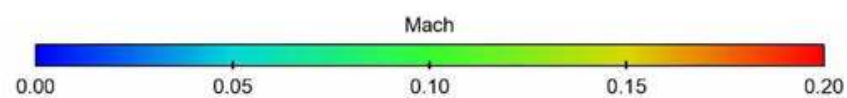

Fig. 20 FOI RANS (TA mean) result of Mach and streamlines at inner left control $\left(\operatorname{cut}\right.$ at $\left.\mathrm{LH}^{\mathrm{H}}\right)$ : $M_{\infty}=0.15$ and $R e=1.6 \cdot 10^{6}$.

If one considers the initial center of rotation as the MRP $(63.5 \%)$, the effect of the center of rotation on pitch damping $\mathrm{Cm}_{q}$ appears quite large, as it could be predicted by simple kinematical consideration: $\mathrm{Cm}_{q}$ at $\mathrm{AOA}=0 \mathrm{deg}$ changes from about -0.31 to -0.50 when the center of rotation moves from $x / c_{R}=63.5$ to $80.9 \%$ (pitching moment is expressed in these calculations at $x / c_{R}=63.5 \%$ ); with the STO-AVT MRP choice (pitching moment expressed at $x / c_{R}=56.6 \%$ ), this variation appears relatively smaller (from -0.48 to -0.56 , when center of rotation moves aft; see Fig. 25) because the increase of $\mathrm{Cm}_{q}$ is compensated by the decrease of $C z_{q}$.

The change of location for the center of rotation has also been numerically assessed. Figure 25 presents a comparison between numerical (dotted lines) and experimental results from rotary balance (solid lines) for the two locations of the center of rotation. It can be seen that, in both cases, the aft location of the center of rotation induces the highest damping effect in pitch.

The comparisons between the computations and experiments show the same tendency, except for the $C z_{q}$ at a high AOA. The pitch damping at $\mathrm{AOA}=10 \mathrm{deg}$ is underpredicted by the numerical simulation. It is an effect of the nonlinear behavior of the pitch dip, which starts at different AOAs between the experiment and the simulations. At a low angle, the simulations are in the linear part of the behavior. At $10 \mathrm{deg}$, the model oscillates between 5 and $15 \mathrm{deg}$, which is above the linear part. At $15 \mathrm{deg}$, the model evolves in a critical area where the aerodynamic variations are large. 


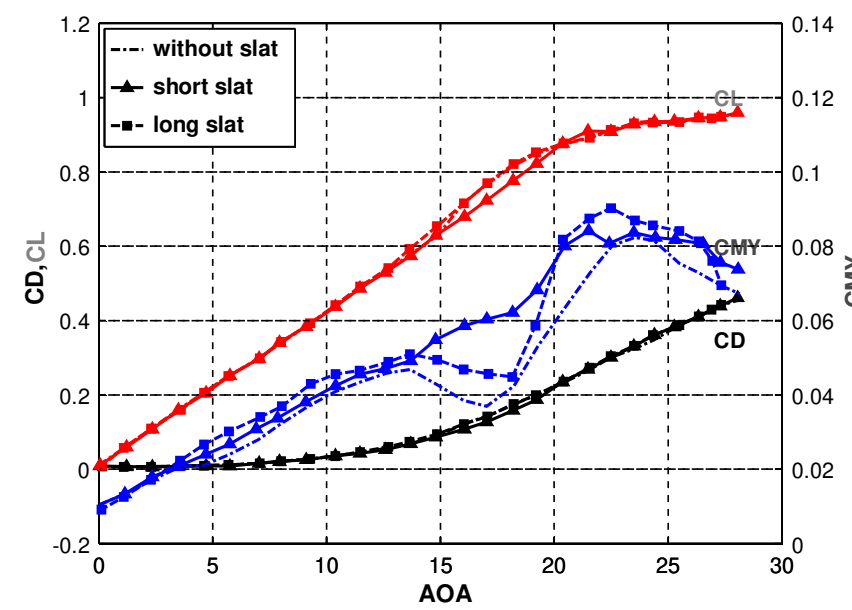

a) Lift, drag, and pitching moment

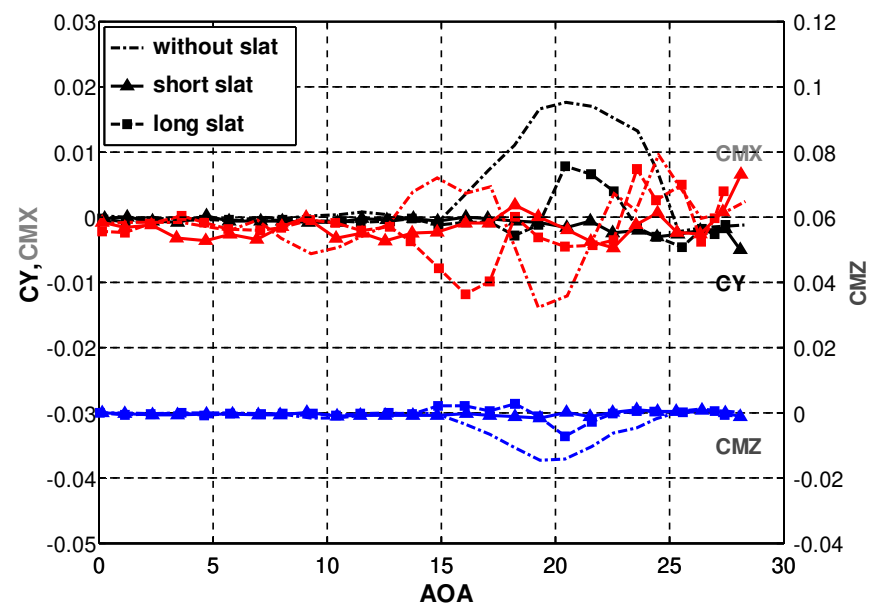

b) Side force, rolling, and yawing moment

Fig. 21 ONERA experimental variation of LE slats length at $x / c=0.326$ with a deflection angle of $-15 \mathrm{deg}, M_{\infty}=0.146$, and $R e=1.13 \cdot 10^{6}$.

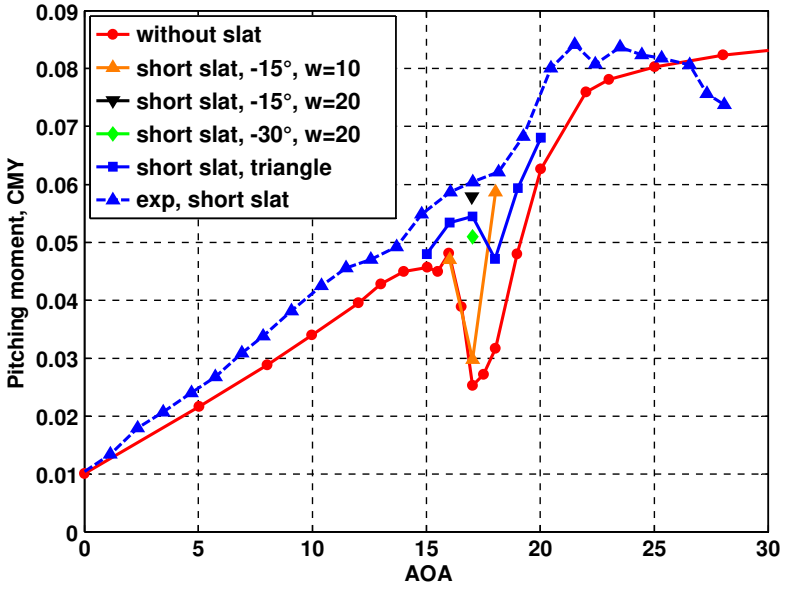

Fig. 22 ONERA numerical pitching moment result from elsA of different LE slat shapes: $M_{\infty}=0.146$ and $R e=1.13 \cdot 10^{6}(\exp$ denotes experiment).

\section{Conclusions}

This paper presents the results of numerical simulations of different control configurations on the 53 deg swept lambda SACCON wing using FOI-developed CFD code Edge and the ONERA-developed solver elsA. The vortical flow at high AOAs results in a nonlinear aerodynamic pitch behavior of the configuration, as seen in experimental tests. The ONERA investigation focuses on unconventional leading-edge slats in order to improve the pitching moment control and lateral instabilities. Dynamic derivatives of the clean configuration are also computed. FOI studies a more conventional trailing-edge control to assess the controllability of the current design.

The initial approach by FOI using steady-state RANS turned out to be numerically unstable due to the highly refined mesh. Therefore, the mean values of force and moment were used, but it was necessary to change to a time-accurate RANS approach to be able to perform postprocessing for flow visualization. Both methods agreed quite well for low angles of attack and were able to capture control surface effects in terms of generating the roll moment. However, the study also revealed problems with the location of trailing-edge controls on the current design with highly swept trailing edges. At higher angles of attack, the crossflow induced by the vortical structures eliminated all control efficiency.

ONERA has performed a mesh refinement study of the clean baseline configuration to assess the convergence and validity of the RANS method. The overall behavior of the aircraft was well predicted by numerical simulations, which compared quite well with experimental data. The major part of the ONERA work was devoted to improve aerodynamic performance by mechanical flow control devices. Different locations, sizes, and shapes of the LE slats were tested. Both experimental measurements and numerical simulations confirmed that the implementation of a leading-edge slat improved the overall aerodynamic performance by changing the topology of the vortical flowfield.

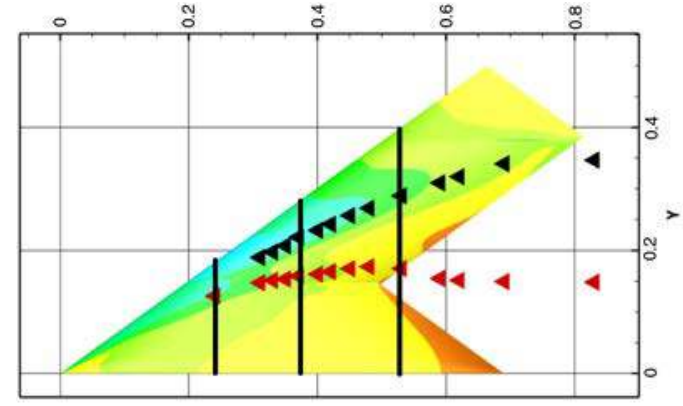

a) No LES, $\mathrm{AOA}=17^{\circ}$

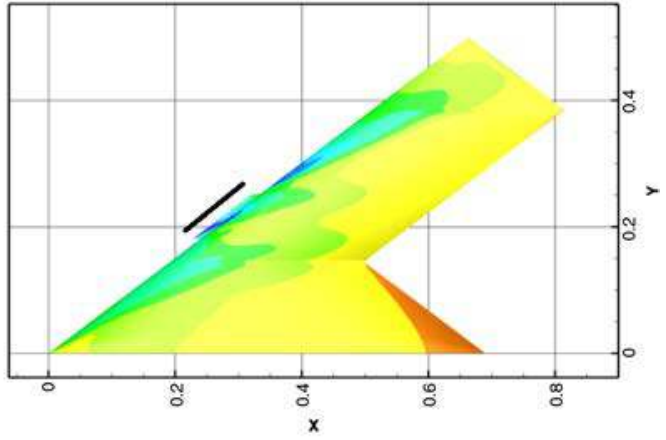

b) Short LES at $\mathrm{x} / \mathrm{c}=0.326, \mathrm{AOA}=17^{\circ}$ 


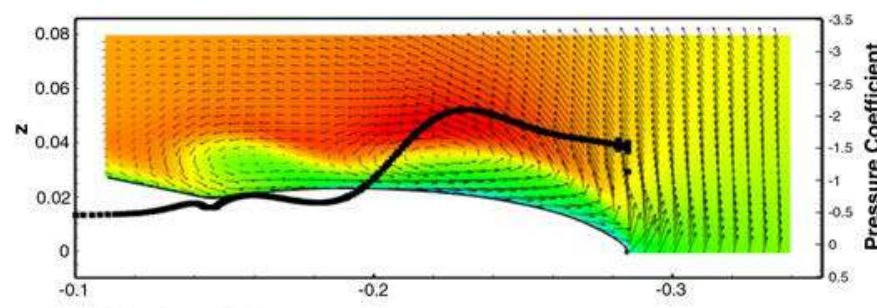

a) No LES, $\mathrm{x} / \mathrm{c}_{\mathrm{r}}=0.55$

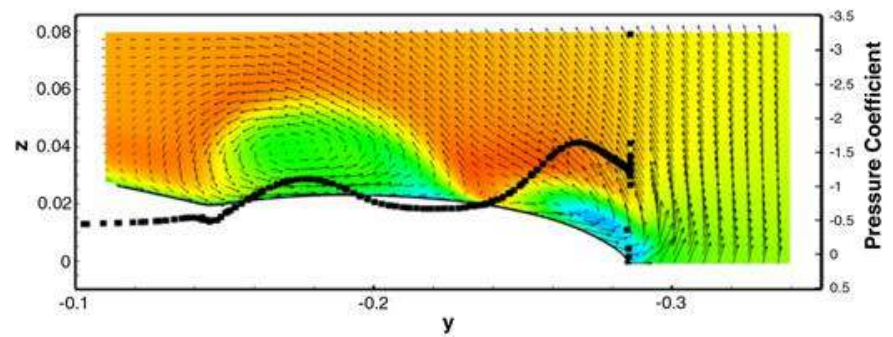

c) With LES, $x / c_{r}=0.55$

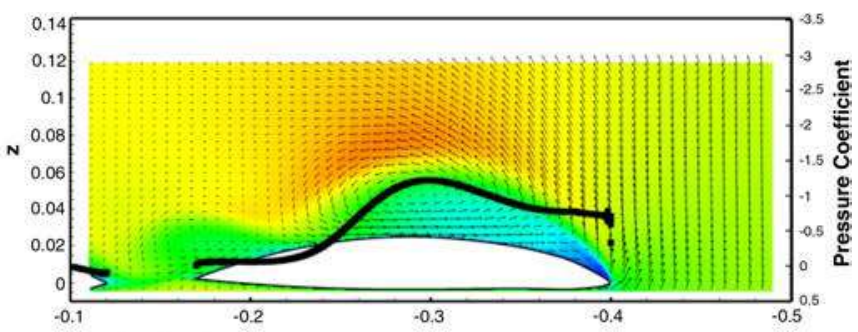

b) No LES, $x / c_{r}=0.77$

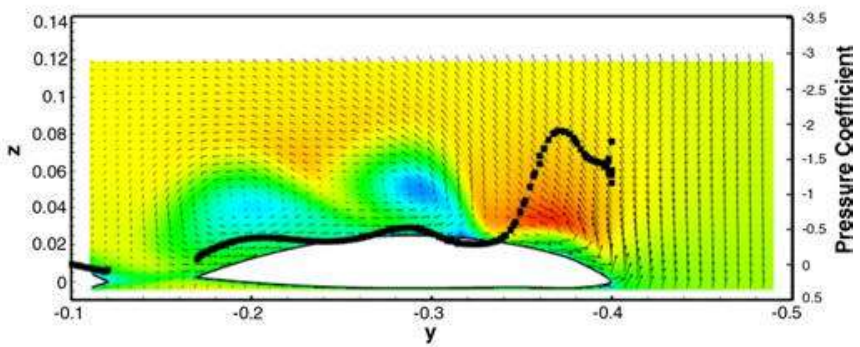

d) With LES, $x / c_{r}=0.77$

Fig. 24 Dimensionless axial velocity at $\mathrm{AOA}=17 \mathrm{deg}$ and $\mathrm{Cp}$ (black line) on the upper surface.
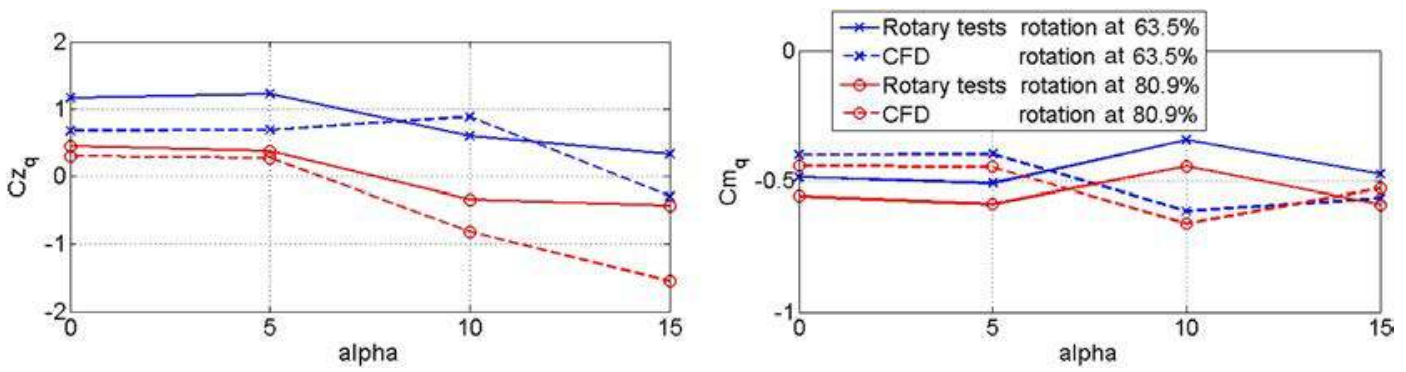

Fig. 25 Comparison between CFD and dynamic tests: effect of center of rotation $\left(x / c_{R}=63.6\right.$ or $\left.80.9 \%\right)$, and moments expressed at the MRP $(56.56 \%)$.

Numerical identification of dynamic derivatives was done by ONERA using a rotating mesh and time-accurate simulations. The results matched well with the dynamic tests from the rotary balance in the vertical tunnel of ONERA. The sensitivity to the location of the center of rotation was demonstrated and led to the conclusion that it was important to superimpose the center of rotation and the MRP during dynamic tests and computations. The dynamic derivatives obtained by simulations showed the same tendency as the experiments.

\section{Acknowledgments}

The first author would like to thank the Swedish Armed Forces for financing the Swedish Defence Research Agency (FOI) research in this Science and Technology Organization/Applied Vehicle Technology-201 task group. The authors would also like to thank all the members of the task group for valuable advice and fruitful discussions. Special thanks go to Andreas Schütte, Kerstin Huber, and Russell M. Cummings.

\section{References}

[1] Drougge, G., "The International Vortex Flow Experiment for Computer Code Validation," ICAS-Proceedings, Vol. 1, 1988, pp. 35-41.

[2] Elsenaar, A., Hjelmberg, L., Bütefisch, K.-A., and Bannink, W. J., "The International Vortex Flow Experiment," AGARD CP437, Vol. 1, 1988, pp. 9-1-9-23.

[3] Hoeijmakers, H. W. M., "Modelling and Numerical Simulation of Vortex Flow in Aerodynamics," AGARD CP-494, 1991, pp. 1-1-1-46.

[4] Hummel, D., "The Second International Vortex Flow Experiment (VFE2): Objectives and First Results," 2nd International Symposium on Integrating CFD and Experiments in Aerodynamics, Cranfield Univ.,
U.K., Sept. 2005; also Journal of Aerospace Engineering, Vol. 220, No. 6, 2006, pp. 559-568.

[5] Hummel, D., "Review of the Second International Vortex Flow Experiment (VFE-2)," 46th AIAA Aerospace Sciences Meeting and Exhibit, AIAA Paper 2008-0377, Jan. 2008.

[6] Schütte, A., and Lüdeke, H., "Numerical Investigations on the VFE 2 65-Degree Rounded Leading Edge Delta Wing Using the Unstructured DLR-TAU-Code," AIAA Paper 2008-0398, 2008.

[7] Lamar, J., and Hummel, D., "Understanding and Modeling Vortical Flows to Improve the Technology Readiness Level for Military Aircraft," RTO/AVT, RTO-TR-AVT-113, Final Rept., 2009.

[8] Schütte, A., Cummings, R. M., Löser, T., and Vicroy, D. D., "Integrated Computational/Experimental Approach to UCAV and Delta-Canard Configurations Regarding Stability and Control," 4th Symposium on Integrating CFD and Experiments in Aerodynamics, von Kármán Inst., Rhode-Saint-Genèse, Belgium, Sept. 2009.

[9] Schütte, A., Hummel, D., and Hitzel, S., "Numerical and Experimental Analyses of the Vortical Flow Around the SACCON Configuration," 28th AIAA Applied Aerodynamics Conference, AIAA Paper 20104690, June-July 2010.

[10] Cummings, R. M., and Schütte, A., "Integrated Computational/ Experimental Approach to Unmanned Combat Air Vehicle Stability and Control Estimation," Journal of Aircraft, Vol. 49, No. 6, 2012, pp. 1542 1557.

[11] Löser, T., "SACCON Static Wind Tunnel Tests at DNW-NWB and $14^{\prime} \times 22^{\prime}$ NASA LaRC," 28th AIAA Applied Aerodynamics Conference, AIAA Paper 2010-4393, June-July 2010.

[12] Vicroy, D., "SACCON Dynamic Wind Tunnel Tests at DNW-NWB and $14^{\prime} \times 22^{\prime}$ NASA LaRC," 28th AIAA Applied Aerodynamics Conference, AIAA Paper 2010-4394, June-July 2010.

[13] Gillot, A., "Static and Dynamic SACCON PIV Tests-Part I: Forward Flowfield," 28th AIAA Applied Aerodynamics Conference, AIAA Paper 2010-4395, June-July 2010. 
[14] Konrath, R., "Static and Dynamic SACCON PIV Tests-Part II: Aft Flow Field," 28th AIAA Applied Aerodynamics Conference, AIAA Paper 2010-4396, June-July 2010.

[15] Tormalm, M., and Schmidt, S., "Computational Study of Static and Dynamic Vortical Flow over the Delta Wing SACCON Configuration Using the FOI Flow Solver Edge," 28th AIAA Applied Aerodynamics Conference, AIAA Paper 2010-4561, June-July 2010.

[16] Frink, N. T., Tormalm, M., and Schmidt, S., "Unstructured CFD Aerodynamic Analysis of a Generic UCAV Configuration," RTO Meeting Proceedings, AC/323(AVT-189)TP/414, RTO-MP-AVT-189, Paper 25, Specialists' Meeting, Portsdown, U.K., Oct. 2011.

[17] Frink, N. T., Tormalm, M., and Schmidt, S., "Three Unstructured Computational Fluid Dynamics Studies on Generic Uninhabited Combat Air Vehicle," Journal of Aircraft, Vol. 49, No 6, 2012, pp.1619-1637.

[18] Morgand, S., "Caractérisation et Contrôle de l'Écoulement Autour d'un Drone Générique," Ph.D. Thesis, UPMC Paris VI, 2013.

[19] Eliasson, P., "Edge, a Navier-Stokes Solver for Unstructured Grids," Proceedings of Finite Volumes for Complex Applications III, 2002, pp. 527-534.

[20] Wallin, S., and Johansson, A. V., "An Explicit Algebraic Reynolds Stress Model of Incompressible and Compressible Flows," Journal of Fluid Mechanics, Vol. 403, Jan. 2000, pp. 89-132.

[21] Hellsten, A., "New Advanced k- $\omega$ Turbulence Model for High-Lift Aerodynamics," AIAA Journal, Vol. 43, No. 9, 2005, pp. 1857-1869.

[22] Cambier, L., Heib, S., and Plot, S., "The Onera elsA CFD Software: Input from Research and Feedback from Industry," Mechanics and Industry, 2013

[23] Le Roy, J.-F., and Morgand, S., "SACCON CFD Static and Dynamic Derivatives Using elsA," 28th AIAA Applied Aerodynamics Conference, AIAA Paper 2010-4562, 2010.
[24] Mialon, B., et al., "Validation of Numerical Prediction of Dynamic Derivatives: The DLR-F12 and the Transcruiser Test Cases," Progress in Aerospace Sciences, Vol. 47, No. 8, Nov. 2011, pp. 674-694.

[25] Tysell, L., "The TRITET Grid Generation System," Proceedings of the 10th ISGG Conference on Numerical Grid Generation, Forth, Crete, Greece 2007 Society of Grid Generation (ISGG), 2007, pp. 13-24.

[26] Gursul, I., Gordnier, R., and Visbal, M., "Unsteady Aerodynamics of Nonslender Delta Wings," Progress in Aerospace Sciences, Vol. 41, Nov. 2005, pp. 515-557.

[27] Riou, J., "Etudes et Contrôle du Décrochage d'Ailes et Gouvernes de Missile en Régime Transsonique," Ph.D. Thesis, Univ. of Paris XIII, France, 2009.

[28] Schütte, A., Hummel, D., and Hitzel, S. M., "Flow Physics Analyses of a Generic Unmanned Combat Aerial Vehicle Configuration," Journal of Aircraft, Vol. 49, No. 6, 2012, pp. 1638-1651.

[29] Jirasek, A., and Cummings, M. R., "SACCON Static and Dynamic Motion Flow Physics Simulations Using COBALT," 29th AIAA Applied Aerodynamics Conference, AIAA Paper 2011-3965, June 2011.

[30] Roosenboom, E. W. M., Konrath, R., Schröder, A., Pallek, D., Otter, D., Morgand, S., Gilliot, A., Monnier, J. C., Le Roy, J. F., Geiler, C., and Pruvost, J., "Stereoscopic Particle Image Velocimetry Flowfield Investigation of an Unmanned Combat Air Vehicle," Journal of Aircraft, Vol. 49, No. 6, 2012, pp. 1584-1596. doi:10.2514/1.C031587.

[31] Le Roy, J.-F., Morgand, S., and Farcy, D., "Static and Dynamic Derivatives on Generic UCAV Without and with Leading Edge Control," 32th AIAA Applied Aerodynamics Conference, AIAA Paper 2014-2391, 2014. 